TRANSACTIONS OF THE

AMERICAN MATHEMATICAL SOCIETY

Volume 354, Number 6, Pages 2417-2459

S 0002-9947(02)02725-3

Article electronically published on February 7, 2002

\title{
ERGODIC AND BERNOULLI PROPERTIES OF ANALYTIC MAPS OF COMPLEX PROJECTIVE SPACE
}

\author{
LORELEI KOSS
}

\begin{abstract}
We examine the measurable ergodic theory of analytic maps $F$ of complex projective space. We focus on two different classes of maps, Ueda maps of $\mathbb{P}^{n}$, and rational maps of the sphere with parabolic orbifold and Julia set equal to the entire sphere. We construct measures which are invariant, ergodic, weak- or strong-mixing, exact, or automorphically Bernoulli with respect to these maps. We discuss topological pressure and measures of maximal entropy $\left(h_{\mu}(F)=h_{\text {top }}(F)=\log (\operatorname{deg} F)\right)$. We find analytic maps of $\mathbb{P}^{1}$ and $\mathbb{P}^{2}$ which are one-sided Bernoulli of maximal entropy, including examples where the maximal entropy measure lies in the smooth measure class. Further, we prove that for any integer $d>1$, there exists a rational map of the sphere which is one-sided Bernoulli of entropy $\log d$ with respect to a smooth measure.
\end{abstract}

\section{INTRODUCTION}

Although the study of complex dynamical systems began at the turn of this century with the work of Fatou, Julia, and Lattès, recent advances in computer technology have contributed to a surge in interest and progress in the field. While there is a vast body of material concerning complex dynamics in one variable, many open questions remain to be answered. Further, there has been less examination of measure-theoretic properties in higher dimensions. Here, we focus on the measurable dynamics of analytic maps of complex projective space.

Two important natural measures for analytic maps $F$ of complex projective space are the measure of maximal entropy $(=\log (\operatorname{deg} F))$ and smooth measure. Freire, Lopez, and Mañé [F-L-M], Mañé [M1], and Lyubich [Ly2] independently proved that every analytic map $f$ of one-dimensional complex projective space $\mathbb{P}^{1}$ has a unique invariant measure of maximal entropy whose support is the Julia set. Further, Mañé [M1] and Lyubich [Ly2 showed that the measure of maximal entropy for an analytic map $f$ of $\mathbb{P}^{1}$ is exact. Freire, Lopez, and Mañé [F-L-M] and Lyubich Ly2 conjectured that, with respect to this unique measure, $f$ is isomorphic to the one-sided $(1 / d, \ldots, 1 / d)$ Bernoulli shift. Mañé [M3] proved that there exists a $k$ such that $f^{k}$ is isomorphic to the one-sided $\left(1 / d^{k}, \ldots, 1 / d^{k}\right)$ Bernoulli shift and hence $f$ is automorphically Bernoulli. For maps of $\mathbb{P}^{n}, n>1$, Fornaess and Sibony [F-S1] and Hubbard and Papadopol [H-P] independently proved that every analytic map $F$ has a measure of maximal entropy.

In contrast to the case of two-sided Bernoulli shifts, there are very few methods by which one can prove the [F-L-M] and [Ly2] conjecture that an analytic map of

Received by the editors March 22, 1999 and, in revised form, March 14, 2000.

2000 Mathematics Subject Classification. Primary 37A25, 37A35, 37F10.

Supported in part by GAANN (Graduate Assistance in Areas of National Need) Fellowship. 
$\mathbb{P}^{1}$ is one-sided Bernoulli of maximal entropy. For example, Ornstein [O] proved that any factor of a two-sided Bernoulli shift is two-sided Bernoulli. It is unknown whether a factor of a one-sided Bernoulli shift must be one-sided Bernoulli. Parry $[\mathrm{P}$ discussed some of the difficulties in a recent paper. Adler, Goodwyn and Weiss A-G-W and Parry $[\mathrm{P}$ proved that a finite extension of a one-sided Bernoulli shift is one-sided Bernoulli. Parry also recently showed that certain skew products with base the one-sided $(1 / 2,1 / 2)$ Bernoulli shift on $X_{2}^{+}$and fiber the circle are isomorphic to the one-sided $(1 / 2,1 / 2)$ Bernoulli shift on $X_{2}^{+}$. In [A-M-T], Ashley, Marcus, and Tuncel give another method of proving that a dynamical system is one-sided Bernoulli. Outside of these examples, the only way to prove that a map is one-sided Bernoulli is to find an independent generating partition that gives an isomorphism. We discuss this technique in Section 2 .

In Section 3, we define a dynamical system called the product-diagonal factor, and we explore the measure-theoretic properties of this construction. Although the theorems proved in this section are, in their own right, of interest in the study of abstract ergodic theory, we apply the results of this chapter to analytic maps of complex projective spaces in Sections 4 and 5 . We show that the properties of nonsingularity, invariance, weak-mixing, strong-mixing, exactness, and automorphically Bernoulli are preserved through the product-diagonal factor construction. Further, we prove that the entropy of the product-diagonal factor map is twice the entropy of the original map. The main result of this section is the following theorem.

Theorem 3.9. If $(X, \mathcal{B}, \mu, f)$ is isomorphic to a one-sided Bernoulli shift of entropy $h$, then the product-diagonal factor is isomorphic to a one-sided Bernoulli shift of entropy $2 h$.

In Section 4 we apply the results of Section 3 to a class of analytic maps of two-dimensional projective space. These maps were first defined by Ueda U1. We define nonsingular measures for Ueda maps and prove that they are productdiagonal factors of analytic maps of $\mathbb{P}^{1}$. Thus, we are able to use the results of Section 3 to obtain measures for analytic maps of $\mathbb{P}^{2}$ with interesting properties such as nonsingularity, invariance, weak- or strong-mixing, exactness, or automorphically Bernoulli. We also prove that the measure-theoretic entropy of the Ueda system is twice the measure-theoretic entropy of the original map of $\mathbb{P}^{1}$. We construct a measure of maximal entropy for Ueda maps, and we provide the first known examples of analytic maps of $\mathbb{P}^{2}$ which are one-sided Bernoulli with respect to a measure of maximal entropy, including examples where this measure lies in the smooth measure class.

In Section 5, we focus on a particular class of rational maps $R$ of the sphere $\mathbb{C}_{\infty}$, those which are critically finite with parabolic orbifold and have a Julia set $J_{R}$ equal to the entire sphere (see $[\mathrm{Be},[\mathrm{E}-\mathrm{L}, \overline{\mathrm{Mi}}]$, and $[\mathrm{D}-\mathrm{H}]$ ). These maps are interesting for a number of reasons. First, they can be described as a semi-conjugation of a toral endomorphism by an elliptic function. This enables us to define a measurable structure on $\mathbb{C}_{\infty}$ making the dynamical system on the sphere a $k$-to-one finite factor of the toral endomorphism.

Second, these maps have been distinguished among all rational maps of the sphere by a theorem of Zdunik [Z]. She showed that critically finite rational maps with parabolic orbifold and $J_{R}=\mathbb{C}_{\infty}$ are the only rational maps for which the 
unique measure of maximal entropy $(=\log (\operatorname{deg} R))$ is equivalent to the smooth measure class.

The main result of Section 5 is Theorem 5.11 where we prove that certain families of rational maps with parabolic orbifold are one-sided Bernoulli with respect to their unique measure of maximal entropy, in this case a smooth measure. This strengthens the result in $\left[\mathrm{B}\right.$ ] and $\left[\mathrm{B}-\mathrm{K}\right.$. that the rational maps $R_{\wp, n}$ and $R_{\wp, n+n i}$ are one-sided Bernoulli of maximal entropy with respect to a smooth probability measure. One consequence of Theorem 5.11 is that for any integer $d>1$, there exists a rational map of the sphere of degree $d$ that is one-sided Bernoulli of entropy $\log d$ with respect to a finite invariant smooth measure. Further, we prove that there exist one-sided Bernoulli maps in each of the four categories of critically finite rational maps with parabolic orbifold and $J_{R}=\mathbb{C}_{\infty}$. The results of Theorem 5.11 prove the [F-L-M] and Ly2 conjecture for some particular cases.

We prove Theorem 5.11 by constructing a direct isomorphism between the rational maps of the sphere and one-sided Bernoulli shifts. The style of our proof has the added benefit that it enables us to understand geometrically how the sets of the partition giving the isomorphism map to each other with respect to each rational map. In [M3], Mañé discusses some of the reasons why a proof of this type is preferable to a more abstract proof.

The results in this article were obtained as part of the author's Ph.D. thesis at the University of North Carolina at Chapel Hill. The author would like to thank her advisor Jane Hawkins for her guidance and suggestions during the preparation of both the thesis and paper. The author also thanks Karl Petersen for helpful comments. The author is grateful to Adam Fitzgerald, Jane Hawkins, and Michael Taylor for help producing the graphics included in this paper.

\section{BACKGROUND}

Let $(X, \mathcal{B}, \mu)$ and $(Y, \mathcal{C}, \nu)$ be Lebesgue probability spaces. We call a measurable map $T$ (backward) nonsingular when $\mu\left(T^{-1} C\right)=0$ if and only if $\nu(C)=0$. We say that $T$ is an endomorphism if $T$ is a measurable map of $(X, \mathcal{B}, \mu)$ to $(X, \mathcal{B}, \mu)$, and $\mu$-almost every point has at most a countable number of preimages.

If an endomorphism $T$ of $(X, \mathcal{B}, \mu)$ is backward nonsingular, Rokhlin [R2] showed that we can replace $X$ with a $T$-invariant subset of full measure and assume that $T$ is forward nonsingular $(\mu(T B)=0$ if and only if $\mu(B)=0)$. Further, Rokhlin proved the existence of a partition $\mathcal{A}$ of $X$ into at most countably many atoms $\mathcal{A}_{1}, \mathcal{A}_{2}, \ldots, \mathcal{A}_{j}, \ldots$ such that each $\mathcal{A}_{j}$ is measurable, $\mu\left(\cup \mathcal{A}_{j}\right)=1, \mu\left(\mathcal{A}_{j}\right)>0$ for all $j$, and $\left.T\right|_{\mathcal{A}_{j}}$ is one-to-one. Further, the $\mathcal{A}_{j}$ 's can be chosen so that each $\mathcal{A}_{j}$ is of maximal measure in $X \backslash \bigcup_{k<j} \mathcal{A}_{k}$ with respect to the property that $\left.T\right|_{\mathcal{A}_{j}}$ is one-to-one. We will call such a partition a Rokhlin partition. If $T$ is forward and backward nonsingular, $\mathcal{A}$ contains $d$ atoms, and $T\left(\mathcal{A}_{j}\right)=X$ for all $j$, then we say that $T$ is a nonsingular d-to-one endomorphism. In the following, we will use the term nonsingular if and only if $T$ is both forward and backward nonsingular.

A nonsingular endomorphism $T$ of $(X, \mathcal{B}, \mu)$ is ergodic if any invariant $B \in \mathcal{B}$ satisfies $\mu(B)=0$ or 1 . We say that $T$ is exact if $\bigcap_{j \geq 0} T^{-j}(\mathcal{B})$ contains only sets of measure 0 or 1 with respect to $\mu$. This is equivalent to $\bigcup_{j \geq 0} T^{-j} T^{j} B$ has $\mu$-measure 0 or 1 for any $B \in \mathcal{B}$. Since every invariant set is in $\bigcap_{j \geq 0} T^{-j}(\mathcal{B})$, if $T$ is exact, then $T$ is ergodic. 
A measure-preserving endomorphism $T$ is weak-mixing if for all $A, B \in \mathcal{B}$,

$$
\lim _{n \rightarrow \infty} \frac{1}{n} \sum_{j=0}^{n-1}\left|\mu\left(T^{-j} A \cap B\right)-\mu(A) \mu(B)\right|=0 .
$$

Suppose $(X, \mathcal{B}, \mu, T)$ and $(Y, \mathcal{C}, \nu, S)$ are nonsingular endomorphisms. A measurable map $g: X \rightarrow Y$ is a factor map if $g \circ T(x)=S \circ g(x)$ for $\mu$-a.e. $x \in X$ and $g$ is surjective $\nu$-a.e. We say that $g$ is a finite factor map if $g$ is finite-to-one $\mu$-a.e. If $(Y, \mathcal{C}, \nu, S)$ is a factor of $(X, \mathcal{B}, \mu, T)$ via the map $g$, we will say that $(Y, \mathcal{C}, \nu, S)$ is a $k$-to-one finite factor if there exist disjoint sets $B_{1}, \ldots, B_{k} \in \mathcal{B}$ such that $\mu\left(\bigcup B_{j}\right)=1$ and, on a set $Y_{1} \subset Y$ with $\nu\left(Y_{1}\right)=1$, every $y \in Y_{1}$ has exactly one preimage $g^{-1}(y) \in B_{j}$ for all $j$.

Let $X_{d}^{+}=\prod_{j=0}^{\infty}\{1,2, \ldots, d\}$ be the collection of one-sided sequences on $d$ symbols. Let $\mathcal{D}$ be the $\sigma$-algebra generated by cylinder sets of $X_{d}^{+}$, and let $\sigma$ be the one-sided shift on $X_{d}^{+}$given by $\sigma\left(x_{0}, x_{1}, x_{2}, \ldots\right)=\left(x_{1}, x_{2}, \ldots\right)$. Consider any vector $\vec{p}=\left(p_{1}, p_{2}, \ldots, p_{d}\right)$ such that $p_{j}>0$ and $\sum_{j=1}^{d} p_{j}=1$. Define a measure $p$ by $p\left(\left[j_{1}, j_{2}, \ldots, j_{k}\right]\right)=p_{j_{1}} p_{j_{2}} \cdots p_{j_{k}}$ where

$$
\left[j_{1}, j_{2}, \ldots, j_{k}\right]=\left\{x \in X_{d}^{+}: x_{l}=j_{1}, x_{l+1}=j_{2}, \ldots, x_{l+k}=j_{k}\right\}
$$

is a cylinder set. We say that a $d$-to-one map $T$ is one-sided Bernoulli with respect to the measure $\mu$ if $(X, \mathcal{B}, \mu, T)$ is isomorphic to $\left(X_{d}^{+}, \mathcal{D}, p, \sigma\right)$ for some vector $\vec{p}$.

Given any endomorphism $(X, \mathcal{B}, \mu, T)$, Rokhlin [R1] defined an invertible transformation called the natural extension of $T$ in the following manner. Let

$$
\tilde{X}=\left\{\tilde{x}=\left(x_{0}, x_{1}, \ldots\right): x_{j} \in X \text { and } T\left(x_{j}\right)=x_{j-1}\right\} .
$$

We define an invertible transformation $\tilde{T}: \tilde{X} \rightarrow \tilde{X}$ by

$$
\tilde{T}(\tilde{x})=\left(T x_{0}, x_{0}, x_{1}, \ldots\right) .
$$

Thus, we associate the invertible map $(\tilde{X}, \mathcal{K}, \tilde{\mu}, \tilde{T})$ to the noninvertible map $(X, \mathcal{B}, \mu, T)$, and we call $(\tilde{X}, \mathcal{K}, \tilde{\mu}, \tilde{T})$ the natural extension of $(X, \mathcal{B}, \mu, T)$. The natural extension is unique up to isomorphism mod 0 (see [R1]). We say that $(X, \mathcal{B}, \mu, T)$ is automorphically Bernoulli if $(\tilde{X}, \mathcal{K}, \tilde{\mu}, \tilde{T})$ is two-sided Bernoulli. We note that many authors call this property weakly Bernoulli, but it was pointed out by M. Smorodinsky that weakly Bernoulli is not a good name since no partition is given in the definition.

The Kolmogorov 0-1 Law implies that one-sided Bernoulli shifts are exact. Further, one-sided Bernoulli shifts are automorphically Bernoulli. However, automorphically Bernoulli and exactness are not related. For example, the product of a two-sided Bernoulli system with a two-to-one map that is one-sided Bernoulli is automorphically Bernoulli but not exact. Further, Kalikow Ka proved that the $T T^{-1}$ map is exact but not automorphically Bernoulli.

Let $\mathcal{P}=\left\{A_{1}, A_{2}, \ldots, A_{d}\right\}$ be a finite partition of $(X, \mathcal{B}, \mu, T)$. We say that $\mathcal{P}$ is independent if for any choice of distinct powers $j_{1}, \ldots, j_{r}$ and for any $A_{j_{k}} \in \mathcal{P}$, $k=1, \ldots, r$, we have

$$
\mu\left(T^{-j_{1}} A_{j_{1}} \cap T^{-j_{2}} A_{j_{2}} \cap \ldots \cap T^{-j_{r}} A_{j_{r}}\right)=\mu\left(A_{j_{1}}\right) \mu\left(A_{j_{2}}\right) \cdots \mu\left(A_{j_{r}}\right) .
$$

Define $\varphi: X \rightarrow X_{d}^{+}$by

$$
\varphi(x)=x_{0}, x_{1}, x_{2}, \ldots \text { where } T^{j}(x) \in A_{x_{j}} .
$$


The map $\varphi$ is the standard way to use a partition to code a system. We define $\mathcal{P}^{k}=\bigvee_{j=0}^{k} T^{-j}(\mathcal{P})$. To show that $(X, \mathcal{B}, \mu, T)$ is one-sided Bernoulli, we could use the following theorem to show that the map $\varphi$ is an isomorphism. A proof of Theorem 2.1 can be found in Petersen $[\mathrm{Pe}]$; see Barnes $\mathrm{B}]$ for the noninvertible case.

Theorem 2.1. Suppose $\mathcal{P}=\left\{A_{1}, A_{2}, \ldots, A_{d}\right\}$ is an independent partition satisfying $\mu\left(A_{j}\right)=p_{j}$. If $\mathcal{P}$ generates under $T$, then the map $\varphi: X \rightarrow X_{d}^{+}$is an isomorphism between $(X, \mathcal{B}, \mu, T)$ and $\left(X_{d}^{+}, \mathcal{D}, p, \sigma\right)$ for the vector $\vec{p}=\left(p_{1}, \ldots, p_{d}\right)$.

Thus we only need to show that $\mathcal{P}$ generates under $T$, i.e. that $\widehat{\bigvee}_{k=0}^{\infty} \mathcal{P}^{k}$ is the Borel $\sigma$-algebra $\mathcal{B}(\mu \bmod 0)$. In general, noninvertible maps are not guaranteed to have a generating partition, so this step is often the most difficult (see [W3]). To show that a partition generates, we could use (for example) the following lemma, whose proof can be found in [M2].

Lemma 2.2. Let $X$ be a compact separable metric space, let $\mathcal{B}$ be the Borel $\sigma$ algebra of $X$, and let $\mu$ be a regular probability measure on $\mathcal{B}$. Suppose $\mathcal{P}^{k}, k \geq 1$, is a sequence of partitions such that

$$
\lim _{k \rightarrow \infty}\left(\sup _{P \in \mathcal{P}^{k}} \operatorname{diam}(P)\right)=0 .
$$

Then ${\widehat{\bigvee_{k=0}^{\infty} \mathcal{P}}}^{k}=\mathcal{B}(\mu \bmod 0)$.

Let $X$ be a compact space with metric $r$, and let $L: X \rightarrow X$ be a continuous transformation. For any natural number $n$ we can define a new metric $r_{n}$ on $X$ by $r_{n}(x, y)=\max _{0 \leq j \leq n-1} r\left(L^{j}(x), L^{j}(y)\right)$. Let $\epsilon>0$. A subset $E$ of $X$ is a $(n, \epsilon)$ separated set with respect to $L$ if $x, y \in E, x \neq y$ implies that $r_{n}(x, y)>\epsilon$. If $g: X \rightarrow \mathbb{R}$ is a continuous function, we let $\left(S_{n} g\right)(x)=\sum_{j=0}^{n-1} g\left(L^{j} x\right)$ for $n \geq 1$. We define

$$
P_{n}(L, g, \epsilon)=\sup \left\{\sum_{x \in E} e^{\left(S_{n} g\right)(x)}: E \text { is a }(n, \epsilon) \text { separated subset of } X\right\} .
$$

Let

$$
P(L, g, \epsilon)=\lim _{n \rightarrow \infty} \sup \frac{1}{n} \log P_{n}(L, g, \epsilon) .
$$

We define the topological pressure of the map $L$ with respect to the function $g$ by $P(L, g)=\lim _{\epsilon \rightarrow 0} P(L, g, \epsilon)$.

A proof of the following variational principle can be found in [W1.

Theorem 2.3. Let $L: X \rightarrow X$ be a continuous map of a compact metric space and let $g: X \rightarrow \mathbb{R}$ be a continuous function. Then

$$
P(L, g)=\sup \left\{h_{\mu}(L)+\int g d \mu\right\}
$$

where the supremum is taken over all invariant probability measures $\mu$ on $(X, \mathcal{B}, L)$.

If there exists a measure $\mu_{g}$ for which the supremum in Theorem 2.3 is attained, then $\mu_{g}$ is called an equilibrium state for $g$. 


\section{The Product Diagonal Factor Map}

In this section, we define a two-point factor of a product space called the productdiagonal factor, and we explore the measure-theoretic properties of this construction. We begin with a nonsingular $d$-to-one endomorphism $\left(X, \mathcal{B}, m_{1}, f\right)$ where $\mathcal{B}$ is a $\sigma$-algebra on $X$ and $m_{1}$ is a nonatomic, nonsingular measure. We define the product map $\hat{f}=f \times f$ on $X \times X$. If we define $m_{2}=m_{1} \times m_{1}$, then $\hat{f}$ is a nonsingular $d^{2}$-to-one endomorphism of $\left(X \times X, \mathcal{B}_{2}=\mathcal{B} \times \mathcal{B}, m_{2}, \hat{f}\right)$.

Let $Z=(X \times X) /(x, y) \sim(y, x)$. Let

$$
\pi: X \times X \rightarrow Z
$$

be defined by

$$
\pi(x, y)=z=\{(x, y),(y, x)\} \in Z .
$$

We can think of the map $\pi$ as folding $X \times X$ along the diagonal. It is clear that the map $F: Z \rightarrow Z$ given by $F=\pi \circ \hat{f} \circ \pi^{-1}$ is well-defined; i.e. the following diagram commutes:

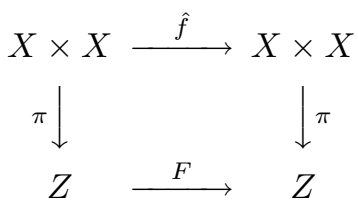

We create a measurable structure on $Z$ by defining $\mathcal{B}_{3}=\left\{E \in Z: \pi^{-1}(E) \in \mathcal{B}_{2}\right\}$. If we define $m_{3}(B)=m_{2}\left(\pi^{-1}(B)\right)$ for all $B \in \mathcal{B}_{3}$, we have defined $F$ to be a factor of $\hat{f}$ with respect to $m_{2}$ and $m_{3}$. We say that $F$ is a product-diagonal factor (or $P-D$ factor $)$ of $f$ when $\left(Z, \mathcal{B}_{3}, m_{3}, F\right)$ is constructed in this manner from the nonsingular endomorphism $\left(X, \mathcal{B}, m_{1}, f\right)$.

Although we are primarily interested in studying the properties of the P-D factor, we will prove the theorems of this section in the greatest generality possible. The proof that any particular property passes to the P-D factor involves showing that the property passes to the product map $\hat{f}$ and then through the factor map $\pi$. Clearly, the properties of nonsingularity, invariance, weak-mixing, and strong-mixing pass from $\left(X, \mathcal{B}, m_{1}, f\right)$ to the P-D factor $\left(Z, \mathcal{B}_{3}, m_{3}, F\right)$.

We next prove that the P-D factor of a $d$-to-one endomorphism is a $d^{2}$-to-one endomorphism.

Proposition 3.1. If $\left(X, \mathcal{B}, m_{1}, f\right)$ is a d-to-one endomorphism and $F$ is a $P$-D factor of $f$, then $\left(Z, \mathcal{B}_{3}, m_{3}, F\right)$ is a $d^{2}$-to-one endomorphism.

Proof. Let $\mathcal{M}=\left\{\mathcal{M}_{1}, \ldots, \mathcal{M}_{d}\right\}$ be a Rokhlin partition of $\left(X, \mathcal{B}, m_{1}, f\right)$. Then $\hat{f}$ is a $d^{2}$-to-one endomorphism using the partition $\hat{\mathcal{M}}=\left\{\bigcup_{k, j=1}^{d} \mathcal{M}_{k} \times \mathcal{M}_{j}\right\}$. We need to modify this partition to obtain a new one which is compatible with the map $\pi$. Let $\Delta=\{(x, x): x \in X\}$ denote the diagonal in $X \times X$. We can find a partition $\gamma^{\prime}=\left\{\gamma_{1}^{\prime}, \gamma_{2}^{\prime}\right\}$ of $X \times X \backslash \Delta$ such that $\gamma_{1}^{\prime}, \gamma_{2}^{\prime} \in \mathcal{B}_{2}, m_{2}\left(\bigcup \gamma_{i}^{\prime}\right)=1,\left.\pi\right|_{\gamma_{i}^{\prime}}$ is one-to-one and onto $Z \backslash \pi(\Delta)$, and $m_{2}\left(\gamma_{i}^{\prime}\right)=\frac{1}{2}$ for $i=1,2$.

Let

$$
\gamma_{1}=\gamma_{1}^{\prime} \cup \Delta \text { and } \gamma_{2}=\gamma_{2}^{\prime} .
$$

Let $\mathcal{M}_{k j}=\mathcal{M}_{k} \times \mathcal{M}_{j}$ and define

$$
\mathcal{N}_{k j}=\left(\mathcal{M}_{k j} \cap \hat{f}^{-1}\left(\gamma_{1}\right)\right) \cup\left(\mathcal{M}_{j k} \cap \hat{f}^{-1}\left(\gamma_{2}\right)\right) .
$$


Then $\mathcal{N}=\left\{\mathcal{N}_{k j}\right\}$ is a Rokhlin partition of $X \times X$, and $\mathcal{K}=\left\{\mathcal{K}_{k j}=\pi\left(\mathcal{N}_{k j}\right)\right\}$ is a Rokhlin partition for $\left(Z, \mathcal{B}_{3}, m_{3}, F\right)$.

The property of exactness passes through the P-D factor construction. Aaronson, Lin, and Weiss $\mathrm{A}-\mathrm{L}-\mathrm{W}$ ] proved (in a more general setting) that the product of two exact maps is exact. Further, any factor of an exact map is exact.

Theorem 3.2. If $\left(X, \mathcal{B}, m_{1}, f\right)$ is exact and $F$ is a $P$-D factor of $f$, then $\left(Z, \mathcal{B}_{3}\right.$, $\left.m_{3}, F\right)$ is exact.

We calculate the measure-theoretic entropy of a P-D factor map $F$ with respect to $m_{3}$ in terms of the measure-theoretic entropy of $f$ with respect to $m_{1}$. Recall that $h_{m_{2}}(\hat{f})=2 h_{m_{1}}(f)$. We use a result of Abramov and Rokhlin A-R to relate the $m_{2}$-entropy of $\hat{f}$ to the $m_{3}$-entropy of $F$ by finding an isomorphism between $\hat{f}$ and a skew product involving $F$. We show that $h_{m_{3}}(F)=2 h_{m_{1}}(f)$ as well. We state this result in Theorem 3.3

Theorem 3.3. If $\left(X, \mathcal{B}, m_{1}, f\right)$ is a measure-preserving transformation and $F$ is a $P$-D factor of $f$, then $h_{m_{3}}(F)=h_{m_{2}}(\hat{f})=2 h_{m_{1}}(f)$.

What remains to be shown is that $h_{m_{3}}(F)=h_{m_{2}}(\hat{f})$. We will actually prove the result for the more general class of finite factor maps. Parry and Tuncel [P-T] gave a proof of Proposition 3.4 in the invertible case. Since their proof assumes the existence of a generator with finite entropy and thus does not immediately pass to the noninvertible case, we include a different proof here for endomorphisms. (See W3 for an example of an endomorphism without a generator with finite entropy.) We say that $g$ is a finite factor map from $(X, \mathcal{B}, \mu, T)$ to $(Y, \mathcal{C}, \nu, S)$ if $g$ is a factor map and there exist disjoint sets $\gamma_{1}, \ldots, \gamma_{k} \in \mathcal{B}$ such that $\mu\left(\bigcup \gamma_{j}\right)=1$ and $g$ is one-to-one on each $\gamma_{j}$. Let $\gamma=\left\{\gamma_{1}, \ldots, \gamma_{k}\right\}$.

Proposition 3.4. If $(Y, \mathcal{C}, \nu, S)$ is a finite factor of $(X, \mathcal{B}, \mu, T)$, then $h_{\mu}(T)=$ $h_{\nu}(S)$.

Let $g: X \rightarrow Y$ be the factor map and $\alpha$ be the point partition on $Y$. We let $\eta$ be the partition given by $g^{-1}(\alpha)$. Elements of this partition consist of $k$ or fewer points. We show that a finite factor satisfies the following lemma.

Lemma 3.5. The partition $\eta$ is invariant and measurable and has $\gamma$ as an independent complement.

Proof. It is clear that $\eta$ is measurable. We have that $\eta$ is invariant (i.e. each element of $T^{-1} \eta$ is the union of elements of $\eta$ ) by the fact that $S=g \circ T \circ g^{-1}$ is well-defined. By definition, $\eta \vee \gamma=\left\{A_{k} \cap B_{j}: A_{k} \in \eta, B_{j} \in \gamma\right\}$ is the point partition. Finally, if $C \in \mathcal{C}$ has positive measure, then $g^{-1} C$ has one branch $B_{j}$ lying in each $\gamma_{j}$. Since $\nu(C)=\mu\left(g^{-1} C\right)=\mu\left(\bigcup_{j=1}^{k}\left(B_{j}\right)\right)$, we have that $\eta$ and $\gamma$ are independent. Finally, $\eta \vee \gamma$ is the partition into points, so $\gamma$ is a complement of $\eta$.

Given an invariant partition $\eta$ with independent complement $\gamma$, Abramov and Rokhlin show that we can find an isomorphism between $X$ and the product of the factor spaces $X / \eta$ and $X / \gamma$,

$$
X \cong X / \eta \times X / \gamma
$$


However, we know that $X / \eta$ is isomorphic to $Y$ and $X / \gamma$ is a $k$ point space. Hence we have

$$
X \cong Y \times\{1, \ldots, k\} .
$$

The measure $\mu_{\eta}$ induced on $X / \eta=Y$ is the measure $\nu$ described previously, and $\mu_{\gamma}$ on $\{1,2, \ldots, k\}$ is the $\left(\mu\left(\gamma_{1}\right), \mu\left(\gamma_{2}\right), \ldots, \mu\left(\gamma_{k}\right)\right)$ measure. We want to find an isomorphism between $T$ on $X$ and a skew product on $Y \times\{1,2, \ldots, k\}$.

We define mappings $V=\left\{V_{y}\right\}$ on $\{1,2, \ldots, k\}$ via the factor map construction as follows. Let $y \in Y$. For any $j$, if $y$ has no preimage under $g$ in $\gamma_{j}$, then let $V_{y}(j)=j$. If $x_{j} \in \gamma_{j}$ is such that $g\left(x_{j}\right)=y$ and $T\left(x_{j}\right) \in \gamma_{l}$, then let $V_{y}(j)=l$. Then $V_{y}:\{1,2, \ldots, k\} \rightarrow\{1,2, \ldots, k\}$ is a well-defined map which we call the fiber map. Thus, following Abramov and Rokhlin, we obtain a skew product on $Y \times\{1,2, \ldots, k\}$ defined by $S \times V_{y}$ which is isomorphic to $T$.

We use the following theorem to calculate the entropy. For an arbitrary partition $\xi$ of $\{1,2, \ldots, k\}$, let

$$
\begin{gathered}
\xi^{j}(y)=\bigvee_{l=0}^{j-1} V_{y}^{-1} V_{S y}^{-1} \ldots V_{S^{l} y}^{-1} \xi, \\
E_{j}(\xi)=\frac{1}{j} \int_{X} H\left(\xi^{j}(z)\right) d \mu_{X}, \\
h_{S}(V, \xi)=\inf _{j} E_{j}(\xi)=\lim _{j \rightarrow \infty} E_{j}(\xi) .
\end{gathered}
$$

The mixed entropy of the skew product with base $S$ and fibers $V=\left\{V_{y}\right\}$ is defined by

$$
h_{S}(V)=\sup _{\xi} h_{S}(V, \xi)
$$

Abramov and Rokhlin proved that if $S \times V_{y}$ is a skew product with base $S$ and fibers $V_{y}(y \in Y)$, then the entropy of $S \times V_{y}$ is given by the formula $h\left(S \times V_{y}\right)=$ $h(S)+h_{S}(V)$. The mixed entropy in our construction is simple to calculate since the fibers consist of only $k$ points. There are only a finite number of partitions $\xi$ of a $k$-point space and hence $E_{j}(\xi) \rightarrow 0$ and $h_{S}(V, \xi)=0$ for any $\xi$. Thus $h_{S}(V)=0$ and $h_{\mu}(T)=h_{\nu}(S)$.

Recall that the partition $\gamma=\left\{\gamma_{1}, \gamma_{2}\right\}$ constructed in equation (1) gives that $\left(Z, \mathcal{B}_{3}, F\right)$ is a two-to-one finite factor of $\left(X \times X, \mathcal{B}_{2}, \hat{f}\right)$. Thus we have completed the proof of Theorem 3.3

Next we prove that if $(X, \mathcal{B}, \mu, T)$ and $(Y, \mathcal{C}, \nu, S)$ are automorphically Bernoulli, then their product is automorphically Bernoulli. Also, we prove that any factor of an automorphically Bernoulli endomorphism is automorphically Bernoulli. As a consequence, the P-D factor of an automorphically Bernoulli endomorphism is automorphically Bernoulli.

Lemma 3.6. If $(X, \mathcal{B}, \mu, T)$ and $(Y, \mathcal{C}, \nu, S)$ are automorphically Bernoulli, then $(X \times Y, \mathcal{B} \times \mathcal{C}, \mu \times \nu, T \times S)$ is automorphically Bernoulli.

Proof. Let $(\tilde{X}, \tilde{\mathcal{B}}, \tilde{\mu}, \tilde{T})$ be the natural extension of $(X, \mathcal{B}, \mu, T)$ and $(\tilde{Y}, \tilde{\mathcal{C}}, \tilde{\nu}, \tilde{S})$ be the natural extension of $(Y, \mathcal{C}, \nu, S)$. Clearly, the natural extension of $(X \times Y, \mathcal{B} \times$ $\mathcal{C}, \mu \times \nu, T \times S)$ is

$$
(\tilde{X} \times \tilde{Y}, \tilde{\mathcal{B}} \times \tilde{\mathcal{C}}, \tilde{\mu} \times \tilde{\nu}, \tilde{T} \times \tilde{S})
$$


Further, we know that a product of two-sided Bernoulli shifts is two-sided Bernoulli, and hence the natural extension of $T \times S$ is two-sided Bernoulli.

Lemma 3.7. Suppose $(Y, \mathcal{C}, \nu, S)$ is a factor of $(X, \mathcal{B}, \mu, T)$. Then if $(X, \mathcal{B}, \mu, T)$ is automorphically Bernoulli, then $(Y, \mathcal{C}, \nu, S)$ is automorphically Bernoulli.

Proof. Let $g: X \rightarrow Y$ be the factor map and thus $\nu=\mu \circ g^{-1}$. The natural extension of $S$, given by $(\tilde{Y}, \tilde{\mathcal{C}}, \tilde{\nu}, \tilde{S})$, is a factor of $(\tilde{X}, \tilde{\mathcal{B}}, \tilde{\mu}, \tilde{T})$. Since factors of twosided Bernoulli shifts are two-sided Bernoulli (see [O]), we obtain that the natural extension of $S$ is two-sided Bernoulli.

Corollary 3.8. If $\left(X, \mathcal{B}, m_{1}, f\right)$ is automorphically Bernoulli and $F$ is a $P$-D factor of $f$, then $\left(Z, \mathcal{B}_{3}, m_{3}, F\right)$ is automorphically Bernoulli.

We now turn to the case when $\left(X, \mathcal{B}, m_{1}, f\right)$ is one-sided Bernoulli.

Theorem 3.9. If $\left(X, \mathcal{B}, m_{1}, f\right)$ is isomorphic to a one-sided Bernoulli shift on d symbols with measure $p$ given by the vector $\vec{p}=\left(p_{1}, p_{2}, \ldots, p_{d}\right)$, and $F$ is a $P-D$ factor of $f$, then $\left(Z, \mathcal{B}_{3}, m_{3}, F\right)$ is isomorphic to a one-sided Bernoulli shift on $d^{2}$ symbols with measure $\hat{p}=p \times p$ given by $\vec{p}=\left(p_{1} p_{1}, p_{1} p_{2}, \ldots, p_{d-1} p_{d}, p_{d} p_{d}\right)$.

Proof. If $\left(X, \mathcal{B}, m_{1}, f\right)$ is isomorphic to a one-sided Bernoulli shift on $d$ symbols with measure $p$ given by $\vec{p}=\left(p_{1}, p_{2}, \ldots, p_{d}\right)$, then $\left(X \times X, \mathcal{B}_{2}, m_{2}, \hat{f}\right)$ is isomorphic to a one-sided Bernoulli shift on $d^{2}$ symbols with measure $\hat{p}=p \times p$ given by $\vec{p}=\left(p_{1} p_{1}, p_{1} p_{2}, \ldots, p_{d-1} p_{d}, p_{d} p_{d}\right)$ (i.e. $\left.\left(X_{d^{2}}^{+}, \mathcal{D}, \hat{p}, \sigma\right)\right)$.

Next, we show that $\left(Z, \mathcal{B}_{3}, m_{3}, F\right)$ is also isomorphic to the one-sided Bernoulli shift $\left(X_{d^{2}}^{+}, \mathcal{D}, \hat{p}, \sigma\right)$. In the invertible case, Ornstein proved that any factor of a twosided Bernoulli shift is a two-sided Bernoulli shift, but we have no such theorem with noninvertible systems. Here, we transfer our investigation of $\hat{f}$ on $X \times X$ to the torus $\mathbb{R}^{2} / \mathbb{Z}^{2}$ and examine the resulting diagonal factor map there.

Let $T:[0,1) \rightarrow[0,1)$ be defined by $T(x)=d x(\bmod 1)$ where $d=\operatorname{deg}(f)$, and let $\mathcal{A}$ be the $\sigma$-algebra of Borel sets on $[0,1)$. Let $\psi: X_{d}^{+} \rightarrow[0,1)$ be defined by

$$
\psi\left(x_{1}, x_{2}, x_{3}, \ldots\right)=\frac{x_{1}}{d}+\frac{x_{2}}{d^{2}}+\frac{x_{3}}{d^{3}} \ldots
$$

The map $\psi$ is one-to-one on $X_{d}^{+} \backslash$ \{eventually constant sequences $\}$ and onto. Furthermore, $T \psi=\psi \sigma$. Let $p$ denote the invariant measure on $X_{d}^{+}$given by $\vec{p}=$ $\left(p_{1}, p_{2}, \ldots, p_{d}\right)$. Then $\mu=p \circ \psi^{-1}$ is a $T$-invariant measure on $([0,1), \mathcal{A})$. Further, $\psi$ gives an isomorphism between $\left(X_{d}^{+}, \mathcal{D}, p, \sigma\right)$ and $([0,1), \mathcal{A}, \mu, T)$. Further, we know that $\left([0,1) \times[0,1), \mathcal{A}_{2}=\mathcal{A} \times \mathcal{A}, \mu_{2}, \hat{T}\right)$ is isomorphic to $\left(X_{d^{2}}^{+}, \mathcal{D}, \hat{p}, \sigma\right)$, where $\mathcal{A}_{2}$ is the $\sigma$-algebra of Borel sets on $[0,1) \times[0,1), \mu_{2}=\mu \times \mu$, and

$$
\hat{T}=\left(\begin{array}{ll}
d & 0 \\
0 & d
\end{array}\right) .
$$

Let $U$ be the period parallelogram given by

$$
U=[0,1) \times[0,1)=\left\{(a, b) \in \mathbb{R}^{2}: 0 \leq a, b<1\right\} .
$$

We define a partition $Q=\left\{B_{j k}: 1 \leq j, k \leq d\right\}$ as follows:

$$
B_{j k}=\left\{(x, y) \in U: \frac{j-1}{d} \leq x<\frac{j}{d} \text { and } \frac{k-1}{d} \leq y<\frac{k}{d}\right\} .
$$

The independent partition $Q$, illustrated in Figure[1, satisfies Theorem 2.1 and gives the isomorphism between $\left([0,1) \times[0,1), \mathcal{A}_{2}=\mathcal{A} \times \mathcal{A}, \mu_{2}, \hat{T}\right)$ and $\left(X_{d^{2}}^{+}, \mathcal{D}, \hat{p}, \sigma\right)$. 


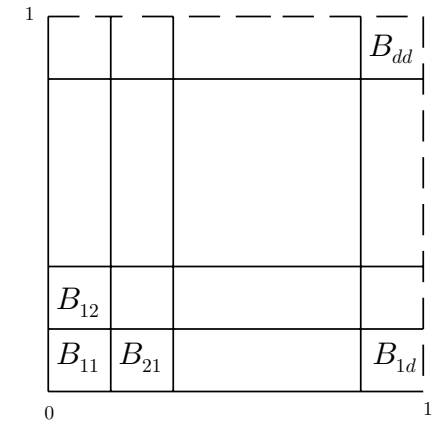

Figure $1 . Q$

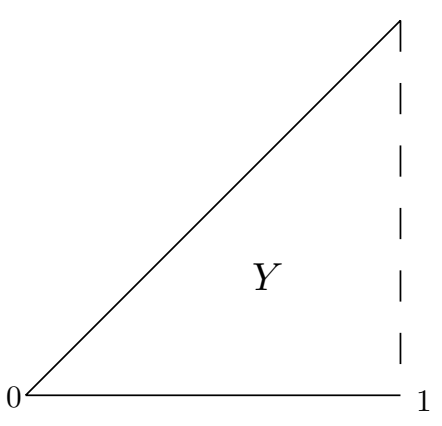

FiguRE 2. $Y$

We construct a factor of $\hat{T}$ via the map $\pi$ which identifies $(x, y) \in[0,1) \times[0,1)$ with $(y, x) \in[0,1) \times[0,1)$. We can represent this factor space by

$$
Y=\{(a, b) \in U: b \leq a\} .
$$

We include an illustration of $Y$ in Figure 2 By the definition of $\mu_{2}$, we note that $\mu_{2}(Y)=1 / 2$. We let $\mathcal{C}$ be the $\sigma$-algebra of Borel sets in $Y$ and define $\mu_{3}(E)=$ $\mu_{2}\left(\pi^{-1}(E)\right)$. We define a map $T_{\pi}: Y \rightarrow Y$ by $T_{\pi}=\pi \circ \hat{T} \circ \pi^{-1}$. Thus we have defined $T_{\pi}$ to be a P-D factor of the map $T$ and we have $\left(Z, \mathcal{B}_{3}, m_{3}, F\right) \cong\left(Y, \mathcal{C}, \mu_{3}, T_{\pi}\right)$.

Proposition 3.10. $\left(Y, \mathcal{C}, \mu_{3}, T_{\pi}\right) \cong\left(X_{d^{2}}^{+}, \mathcal{D}, \hat{p}, \sigma\right)$.

We need to modify the partition $Q$ to make it compatible with the factor map $\pi$. We define a partition $\mathcal{P}=\left\{A_{1}, \ldots, A_{d^{2}}\right\}$ of $Y$ by

$$
\begin{aligned}
& A_{k j}=\left\{\begin{array}{c}
(x, y) \in Y: \frac{k-1}{d} \leq x \leq \frac{k}{d}, \frac{j-1}{d} \leq y \leq \frac{j}{d}, \\
\text { and } y<x-\frac{k-j}{d} \text { where } j \leq k
\end{array}\right\}, \\
& A_{j k}=\left\{\begin{array}{l}
(x, y) \in Y: \frac{k-1}{d} \leq x \leq \frac{k}{d}, \frac{j-1}{d} \leq y \leq \frac{j}{d}, \\
\text { and } y>x-\frac{k-j}{d} \text { where } j<k
\end{array}\right\} .
\end{aligned}
$$

The partition $\mathcal{P}$ in the case where $d=2$ is illustrated in Figure 3. We also include an illustration of the extension of $\mathcal{P}$ to $U$ using the identification of $(x, y)$ with $(y, x)$ in Figure 4. Since $\mu_{2}$ is the product measure $\mu \times \mu$, we have that $\mu_{2}$ is symmetric about the diagonal and $\mu_{3}\left(A_{k j}\right)=\mu_{2}\left(B_{k j}\right)$. Further, $\mathcal{P}$ is independent. Define $\varphi: Y \rightarrow X_{d^{2}}^{+}$by

$$
\varphi(x, y)=x_{0} y_{0}, x_{1} y_{1}, x_{2} y_{2}, \ldots \text { where } T_{\pi}^{j}(x, y) \in A_{x_{j} y_{j}} .
$$

Notice that $T_{\pi}^{j}\left(T_{\pi}(x, y)\right)=T_{\pi}^{j+1}(x, y) \in A_{x_{j+1} y_{j+1}}$. Therefore, $\sigma \varphi(x, y)=$ $\varphi\left(T_{\pi}(x, y)\right)$.

We define $\mathcal{P}^{k}=\bigvee_{j=0}^{k} T_{\pi}^{-j}(\mathcal{P})$. We need to show that $\mathcal{P}$ is a generating partition,

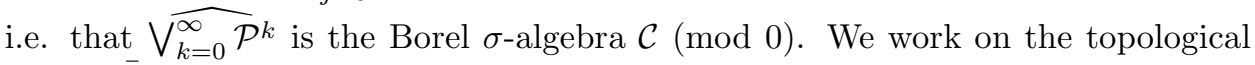
closure $\bar{Y}$ of $Y$ with the standard flat Euclidean metric. If we restrict the Euclidean metric to $\bar{Y}$ we see that $\bar{Y}$ is a locally complete separable metric space. We illustrate $\mathcal{P}^{1}$ in the case where $d=2$ in Figure [5] Each region has its own name. 


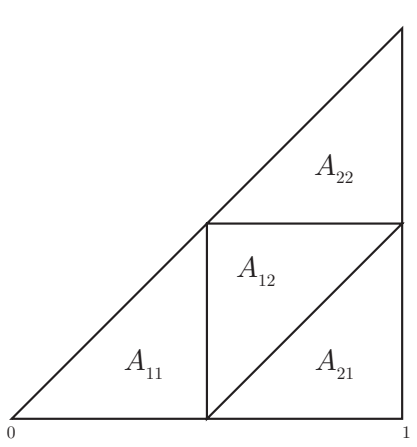

FiguRE 3. $\mathcal{P}$

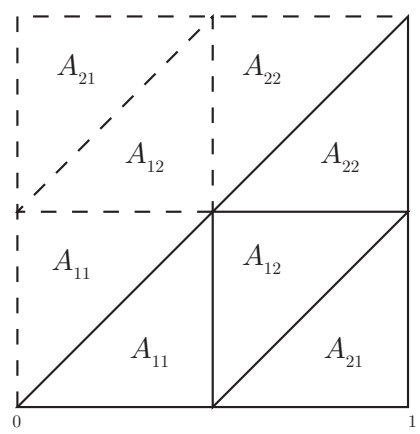

Figure 4. $\mathcal{P}$ extended to $U$

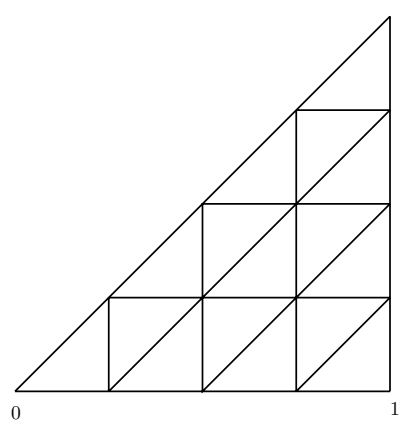

FiguRE $5 . \mathcal{P}^{1}=\mathcal{P} \vee T^{-1} \mathcal{P}$

When $d=2$, we see that $\mathcal{P}^{k}=\bigvee_{j=0}^{k} T_{\pi}^{-j}(\mathcal{P})$ cuts every $P \in \mathcal{P}^{k-1}$ into 4 isosceles triangles of diameter $\frac{\sqrt{2}}{2^{k+1}}$. Therefore,

$$
\lim _{k \rightarrow \infty}\left(\sup _{P \in \mathcal{P}^{k}} \operatorname{diam}(P)\right)=\lim _{k \rightarrow \infty} \frac{\sqrt{2}}{2^{k+1}}=0 .
$$

In the degree $d$ case, $\mathcal{P}^{k}=\bigvee_{j=0}^{k} T_{\pi}^{-j}(\mathcal{P})$ cuts every $P \in \mathcal{P}^{k-1}$ into $d^{2}$ isosceles triangles of diameter $\frac{\sqrt{2}}{d^{k+1}}$. Therefore,

$$
\lim _{k \rightarrow \infty}\left(\sup _{P \in \mathcal{P}^{k}} \operatorname{diam}(P)\right)=\lim _{k \rightarrow \infty} \frac{\sqrt{2}}{d^{k+1}}=0 .
$$

Applying Theorem 2.1 and Lemma 2.2, $\varphi$ is an isomorphism. We have completed the proof of Proposition 3.10

We have shown in this section that many measure-theoretic properties will pass from a dynamical system to the P-D factor map. However, there are properties that fail to be preserved by this construction. For example, let $X=\{a, b\}$ and $\mu(a)=\mu(b)=1 / 2$. If $T(a)=b$ and $T(b)=a$, then $(X, \mathcal{B}, \mu, T)$ is ergodic, but the P-D factor will not be ergodic. 
We also give a specific example of an ergodic measure for a map of the interval for which the resulting measure on the P-D factor is not ergodic. This example will be applied to analytic maps of $\mathbb{P}^{2}$ later in this paper.

Proposition 3.11. Let $T:[0,1) \rightarrow[0,1)$ be defined by $T(x)=d x(\bmod 1)$ and let $\mathcal{A}$ be the $\sigma$-algebra of Borel sets on $[0,1)$. Then there exists an ergodic measure $\nu$ on $([0,1), \mathcal{A}, T)$ such that the $P-D$ factor is not ergodic.

Proof. Eigen and Hawkins [E-H] constructed an ergodic, not weak-mixing measure $\mu$ on the $d$-shift on $X_{d}^{+}$. They accomplished this by constructing $\mu$ so that $\left(X_{d}^{+}, \mathcal{D}, \mu, \sigma\right)$ has the odometer with its unique invariant measure $\rho$ as a maximal automorphic factor. Since the odometer is not weak-mixing with respect to $\rho, \mu$ cannot be weak-mixing.

We define a point map $\psi: X_{d}^{+} \rightarrow[0,1)$ as in eq. (2). We use $\psi$ to transfer the nonatomic Eigen-Hawkins measure $\mu$ to $[0,1)$ by letting $\nu=\mu \psi^{-1}$. Thus $([0,1), \mathcal{A}, \nu, T)$ is ergodic and not weak-mixing, and $\left(X_{d^{2}}^{+}, \mathcal{D} \times \mathcal{D}, \mu_{2}=\mu \times \mu, \sigma\right) \cong$ $\left([0,1) \times[0,1), \mathcal{A}_{2}, \nu_{2}=\nu \times \nu, \hat{T}\right)$ is not ergodic. In eq. (4) we defined a point map $\phi: Y \rightarrow X_{d^{2}}^{+}$where $Y$ is defined in eq. (3). Define $\nu_{3}=\nu_{2} \pi^{-1}=(\nu \times \nu) \pi^{-1}$ so that $\left(Y, \mathcal{C}, \nu_{3}, T_{\pi}\right)$ is a P-D factor of $\left([0,1) \times[0,1), \mathcal{A}_{2}, \nu_{2}, \hat{T}\right)$. Then Theorem 2.1] and Lemma 2.2 hold for these measures as well and thus we obtain that $\left(X_{d^{2}}^{+}, \mathcal{D} \times\right.$ $\left.\mathcal{D}, \mu_{2}, \sigma\right) \cong\left(Y, \mathcal{C}, \nu_{3}, T_{\pi}\right)$, and the $\mathrm{P}-\mathrm{D}$ factor is not ergodic.

Next, we apply the results of this section to a family of analytic maps of complex projective space.

\section{Analytic Maps of Complex Projective Space}

Let $\mathbb{P}^{n}$ denote $n$-dimensional complex projective space. A holomorphic map $F$ of $\mathbb{P}^{n}$ is given in homogeneous coordinates by

$$
F\left(\left[x_{0}, \ldots, x_{n}\right]\right)=\left[p_{0}\left[x_{0}, \ldots, x_{n}\right], \ldots, p_{n}\left[x_{0}, \ldots, x_{n}\right]\right]
$$

where each $p_{j}$ is a homogeneous polynomial of degree $d$ (i.e. $p_{j}(\lambda x)=\lambda^{d} p_{j}(x)$ ), and there is no factor common to all of the $p_{j}$ 's. We define the Fatou Set of $F$ by

$$
\begin{aligned}
\Omega_{F}=\left\{x=\left[x_{0}, \ldots, x_{n}\right] \in \mathbb{P}^{n}:\right. & \text { there exists a neighborhood } V \text { of } x \\
& \text { such that } \left.\left\{\left.F^{k}\right|_{V}\right\}_{k \geq 0} \text { forms a normal family }\right\} .
\end{aligned}
$$

The Julia Set of $F$ is $J_{F}=\mathbb{P}^{n}-\Omega_{F}$.

In [U1], Ueda studied analytic maps of $\mathbb{P}^{n}$ constructed in the following manner. For $n>1$, there exist quotient maps $\pi_{n}$ from the product of $n$ copies of $\mathbb{P}^{1}$ to $\mathbb{P}^{n}$,

$$
\pi_{n}: \mathbb{P}^{1} \times \ldots \times \mathbb{P}^{1} \rightarrow \mathbb{P}^{n}
$$

Points in $\mathbb{P}^{1} \times \ldots \times \mathbb{P}^{1}$ are identified through a group action via the symmetric group on $n$ letters. The degree of the map $\pi_{n}$ is therefore $n$ !, and the branch locus of the map $\pi_{n}$ can be described by

$$
\Delta_{n}=\left\{\left(a_{1}, a_{2}, \ldots, a_{n}\right) \in \mathbb{P}^{1} \times \ldots \times \mathbb{P}^{1} \mid a_{j}=a_{k} \text { for some } j \neq k\right\} .
$$

If $f: \mathbb{P}^{1} \rightarrow \mathbb{P}^{1}$ is analytic, then define

$$
\hat{f}=f \times \ldots \times f: \mathbb{P}^{1} \times \ldots \times \mathbb{P}^{1} \rightarrow \mathbb{P}^{1} \times \ldots \times \mathbb{P}^{1} .
$$


We thus obtain a well-defined map $F_{n}: \mathbb{P}^{n} \rightarrow \mathbb{P}^{n}$ given by the following commutative diagram:

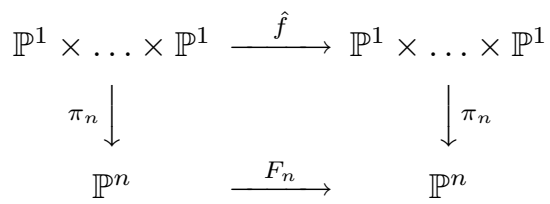

We call a map constructed in the above fashion a Ueda map.

In [U1], Ueda proved the following proposition.

Proposition 4.1 ([U1]). If $f$ is a holomorphic map of $\mathbb{P}^{1}$ and $\hat{f}=f \times f$ and $F_{2}$ are given by Ueda's construction, then

(1) $J_{\hat{f}}=\left(J_{f} \times \mathbb{P}^{1}\right) \cup\left(\mathbb{P}^{1} \times J_{f}\right), J_{F_{2}}=\pi_{2}\left(J_{\hat{f}}\right)$,

(2) $J_{f}=\mathbb{P}^{1} \Leftrightarrow J_{\hat{f}}=\mathbb{P}^{1} \times \mathbb{P}^{1} \Leftrightarrow J_{F_{2}}=\mathbb{P}^{2}$.

For example, if $n=2$ we can define

$$
\pi_{2}\left([a, b],\left[a^{\prime}, b^{\prime}\right]\right)=\left[a a^{\prime}+b b^{\prime}, b b^{\prime}-a a^{\prime}, a b^{\prime}+a^{\prime} b\right] .
$$

Example 4.2 was given in [U1].

Example 4.2. Let $R: \mathbb{C}_{\infty} \rightarrow \mathbb{C}_{\infty}$ be given by

$$
R(z)=\frac{1}{2 i}\left(z-\frac{1}{z}\right)
$$

Then $R$ can be represented as a map $f$ of $\mathbb{P}^{1}$ by $f([a, b])=\left[2 i a b, b^{2}-a^{2}\right]$. Therefore,

$$
\hat{f}\left([a, b],\left[a_{1}, b_{1}\right]\right)=\left(\left[2 i a b, b^{2}-a^{2}\right],\left[2 i a_{1} b_{1}, b_{1}{ }^{2}-a_{1}{ }^{2}\right]\right) .
$$

Using $\pi_{2}$ defined in eq. (5), we obtain a holomorphic map of $\mathbb{P}^{2}$ given by

$$
F_{2}([x, y, z])=\left[y^{2}-z^{2}, 2 x^{2}-y^{2}-z^{2}, 2 i y z\right] .
$$

Since all critical points of $R$ are preperiodic but not periodic, $J_{R}=\mathbb{C}_{\infty}$. Proposition 4.1 implies that $J_{F_{2}}=\mathbb{P}^{2}$.

4.1. Measurable Properties. Let $\widehat{\mathcal{B}}=\mathcal{B} \times \ldots \times \mathcal{B}$. Let $m_{1}$ be a nonatomic, nonsingular measure for $f$ on $\mathbb{P}^{1}$, and let $m_{n}=m_{1} \times \ldots \times m_{1}$. Let $\mathcal{B}_{n}=\{E \in$ $\mathbb{P}^{n}: \pi_{n}^{-1}(E) \in \widehat{\mathcal{B}}$ and define $m_{n, \pi}=m_{n} \pi_{n}^{-1}$. Then $F_{n}$ is a $n !$-to-one finite factor of $\hat{f}=f \times \ldots \times f$. We thus have the following corollaries of Section 3

Corollary 4.3. Suppose $\left(\mathbb{P}^{1}, \mathcal{B}, m_{1}, f\right)$ has any of the following properties and $F_{n}$ is a Ueda map:

1. nonsingular,

2. measure-preserving,

3. weak-mixing,

4. strong-mixing,

5. exact,

6. automorphically Bernoulli.

Then $\left(\mathbb{P}^{n}, \mathcal{B}_{n}, m_{n, \pi}, F_{n}\right)$ has the same property.

Corollary 4.4. $h_{m_{n, \pi}}\left(F_{n}\right)=h_{m_{n}}(\hat{f})=n h_{m_{1}}(f)$.

We note that the following two corollaries only hold for the case $n=2$. 
Corollary 4.5. If $f$ is a d-to-one endomorphism with respect to $m_{1}$ and $F$ is a Ueda map, then $\left(\mathbb{P}^{2}, \mathcal{B}_{2}, m_{2, \pi}, F_{2}\right)$ is a $d^{2}$-to-one endomorphism.

Corollary 4.6. Suppose $\left(\mathbb{P}^{1}, \mathcal{B}, m_{1}, f\right)$ is one-sided Bernoulli. Then the Ueda system $\left(\mathbb{P}^{2}, \mathcal{B}_{2}, m_{2, \pi}, F_{2}\right)$ is one-sided Bernoulli.

4.2. Lebesgue Measure Zero. For an analytic map $f$ of $\mathbb{P}^{1}$ it is conjectured that either $J_{f}=\mathbb{P}^{1}$ or the Julia set has measure zero with respect to normalized surface area measure $m_{l}$ (see $[\mathrm{C}-\mathrm{G}]$ ). (We will call any element of the smooth measure class Lebesgue measure unless a specific representative is relevant.) In fact, there are many conditions under which analytic maps of $\mathbb{P}^{1}$ are known to have Julia sets with zero Lebesgue measure. We refer the reader to [C-G] for a more detailed discussion. The proof of the following proposition is straightforward.

Proposition 4.7. Let $\left(\mathbb{P}^{1}, \mathcal{B}, m_{1}, f\right)$ be a holomorphic map of $\mathbb{P}^{1}$ and let $F_{n}$ be a Ueda map. Then $m_{1}\left(J_{f}\right)=0$ if and only if $m_{n}\left(J_{F_{n}}\right)=0$.

Example 4.8. The rational map of the sphere $R(z)=z^{2}$ is expanding on the Julia set $J_{R}=\{|z|=1\}$, and thus $m_{l}\left(J_{R}\right)=0$. Using Ueda's construction, we obtain a map of $\mathbb{P}^{2}$ given by $F_{2}([x, y, z])=\left[\frac{x^{2}+y^{2}}{2}, x y, \frac{y^{2}-x^{2}}{2}+z^{2}\right]$. Proposition 4.7 implies that the Lebesgue measure of $J_{F_{2}}$ is zero.

4.3. Measure of Maximal Entropy. Another interesting measure in complex dynamics is the measure of maximal entropy. In [G], Gromov proved if $G$ is a holomorphic map of $\mathbb{P}^{n}$, then $h_{t o p}(G)=\log (\operatorname{deg} G)$. Freire, Lopez, and Mañé [F-L-M], Mañé [M1], and Lyubich [Ly2 independently proved that every analytic map of $\mathbb{P}^{1}$ has a unique invariant measure of maximal entropy whose support is the Julia set.

Independently, Fornaess and Sibony [F-S2] and Hubbard and Papadopol [H-P] proved if $G$ is any holomorphic map of $\mathbb{P}^{2}$, then $G$ has a measure of maximal entropy $\mu$, and $\operatorname{supp}(\mu) \subseteq J_{G}$. In each paper the authors are unable to determine exactly where the support of this measure lies. Further, it is unknown whether holomorphic maps of $\mathbb{P}^{2}$ can have more than one measure of maximal entropy.

We can use the Ueda construction to obtain a measure of maximal entropy on $\mathbb{P}^{n}$ and determine explicitly the support of this measure. We state these results in the following theorem.

Theorem 4.9. If $f$ is a holomorphic map of $\mathbb{P}^{1}$ with $m_{1}$ the invariant measure of maximal entropy and $\left(\mathbb{P}^{n}, \mathcal{B}_{n}, m_{n, \pi}, F_{n}\right)$ is a Ueda map, then $m_{n, \pi}$ is a measure of maximal entropy for $F_{n}$ which is supported on $\pi\left(J_{f} \times \ldots \times J_{f}\right) \subseteq J_{F}$. If $J_{f} \neq \mathbb{P}^{1}$, then $\operatorname{supp}\left(m_{n, \pi}\right) \subsetneq J_{F_{n}}$. Further, $F_{n}$ is exact and automorphically Bernoulli with respect to $m_{n, \pi}$.

Proof. The fact that $m_{n, \pi}$ has maximal entropy follows immediately from Corollary 4.4. It is clear that $\operatorname{supp}\left(m_{n, \pi}\right)=\pi_{n}\left(\operatorname{supp}\left(m_{n}\right)\right)$. Therefore we have $\operatorname{supp}\left(m_{n, \pi}\right)=$ $\pi_{n}\left(J_{f} \times \ldots \times J_{f}\right) \subseteq J_{F}$. If $J_{f} \neq \mathbb{P}^{1}$, then $J_{f} \times \ldots \times J_{f} \subsetneq J_{\hat{f}}$ and thus $\operatorname{supp}\left(m_{n, \pi}\right)=$ $\pi_{n}\left(J_{f} \times \ldots \times J_{f}\right) \subsetneq J_{F}$.

The manner in which $m_{n, \pi}$ is constructed leads us to the following conjecture, which follows immediately from Corollary 4.6 and the conjecture in [F-L-M] and Ly2.

Conjecture. If $f$ is a holomorphic map of $\mathbb{P}^{1}$ with $m_{1}$ the invariant measure of maximal entropy and $\left(\mathbb{P}^{2}, \mathcal{B}_{2}, m_{2, \pi}, F_{2}\right)$ is a Ueda map, then $\left(\mathbb{P}^{2}, \mathcal{B}_{2}, m_{2, \pi}, F_{2}\right)$ is isomorphic to a one-sided Bernoulli shift of entropy $\log \left(\operatorname{deg} F_{2}\right)$. 
4.4. Topological Pressure and Equilibrium States. Lyubich Ly2 proved that an equilibrium state exists for any analytic map $f: \mathbb{P}^{1} \rightarrow \mathbb{P}^{1}$ and any continuous function $g: \mathbb{P}^{1} \rightarrow \mathbb{R}$. We are interested in describing how topological pressure changes through Ueda's construction and determining when we can find equilibrium states. We begin with the following theorem.

Theorem 4.10. Let $f$ be a holomorphic map of $\mathbb{P}^{1}$, let $g: \mathbb{P}^{1} \rightarrow \mathbb{R}$ be a continuous function, and let $F_{2}$ be the Ueda map constructed from $f$. Then $P\left(F_{2}, j\right)=$ $P(\hat{f}, \hat{g})=2 P(f, g)$, where $\hat{g}: \mathbb{P}^{1} \times \mathbb{P}^{1} \rightarrow \mathbb{R}$ is defined by $\hat{g}(a, b)=g(a)+g(b)$, and $j: \mathbb{P}^{2} \rightarrow \mathbb{R}$ is defined by $j([x, y, z])=2 \hat{g} \circ \pi_{2}^{-1}([x, y, z])$.

Proof. See [W1] for a proof that $P(\hat{f}, \hat{g})=2 P(f, g)$. Recall the definition of $\gamma_{1}$ in eq. (1). Clearly, $j=2 \hat{g} \circ \pi_{2}^{-1}$ is continuous. Recall from Corollary 4.4 that for any invariant measure $m_{2}$ on $\mathbb{P}^{1} \times \mathbb{P}^{1}$ and $m_{2, \pi}=m_{2} \pi_{2}^{-1}$ on $\mathbb{P}^{2}$, we have

$$
h_{m_{2}}(\hat{f})=h_{m_{2, \pi}}\left(F_{2}\right) .
$$

By definition, the invariant measure $m_{2, \pi}$ is constructed from the invariant measure $m_{2}$. Further, we can lift any invariant measure $\mu$ on $\mathbb{P}^{2}$ to an invariant measure $\mu_{*}$ on $\mathbb{P}^{1} \times \mathbb{P}^{1}$ by defining

$$
\mu_{*}(B)=\frac{\mu\left(\pi_{2}\left(B \cap \gamma_{1}\right)\right)}{2}+\frac{\mu\left(\pi_{2}\left(B \cap \gamma_{2}\right)\right)}{2} .
$$

Thus every invariant $m_{2}$ is associated with an invariant $m_{2, \pi}$ and vice versa. Then

$$
\begin{aligned}
& \int_{\mathbb{P}^{1} \times \mathbb{P}^{1}} \hat{g} d m_{2}=\int_{\gamma_{1}} \hat{g} d m_{2}+\int_{\gamma_{2}} \hat{g} d m_{2}=\int_{\gamma_{1}} \hat{g} \pi_{2}^{-1} \pi_{2} d m_{2}+\int_{\gamma_{2}} \hat{g} \pi_{2}^{-1} \pi_{2} d m_{2} \\
& =\int_{\mathbb{P}^{2}} \hat{g} \pi_{2}^{-1} d m_{2, \pi}+\int_{\mathbb{P}^{2}} \hat{g} \pi_{2}^{-1} d m_{2, \pi}=2 \int_{\mathbb{P}^{2}} \hat{g} \pi_{2}^{-1} d m_{2, \pi}=\int_{\mathbb{P}^{2}} j d m_{2, \pi} .
\end{aligned}
$$

Therefore,

$$
P\left(F_{2}, j\right)=\sup \left\{h_{m_{2, \pi}}\left(F_{2}\right)+\int j d m_{2, \pi}\right\}=\sup \left\{h_{m_{2}}(\hat{f})+\int \hat{g} d m_{2}\right\}=P(\hat{f}, \hat{g}) .
$$

We have completed the proof of Theorem 4.10

Theorem 4.11. Let $f$ be an analytic map of $\mathbb{P}^{1}$ and let $g: \mathbb{P}^{1} \rightarrow \mathbb{R}$ be a continuous function. If $m_{1}$ is an equilibrium state for $g$, then $m_{2}$ is an equilibrium state for $\hat{g}$ and $m_{2, \pi}$ is an equilibrium state for $j$.

Proof. The variational principle (Theorem 2.3) implies that $P(\hat{f}, \hat{g}) \geq h_{m_{2}}(\hat{f})+$ $\int \hat{g} d m_{2}$. We will show that, in fact, equality is obtained and thus $m_{2}$ is an equilibrium state for $\hat{g}$. Recall from Corollary 4.4 that $h_{m_{2}}(\hat{f})=2 h_{m_{1}}(f)$. Fubini's Theorem implies that $\int \hat{g} d m_{2}=2 \int g d m_{1}$. Using Theorem 4.10 we have $P(\hat{f}, \hat{g})=2 P(f, g)=2\left(h_{m_{1}}(f)+\int g d m_{1}\right)=h_{m_{2}}(\hat{f})+\int \hat{g} d m_{2}$. Thus $m_{2}$ is an equilibrium state for $\hat{g}$.

Since $m_{1}$ is an invariant probability measure, we know that $m_{2, \pi}$ is an invariant probability measure by Corollary 4.3. The proof of Theorem 4.10 gives that $m_{2, \pi}$ is an equilibrium state for $j$. 
4.5. Acip, Type III, and Ergodic but Not Weak-Mixing Examples. We conclude this section with some interesting examples that arise through this construction.

Example 4.12 (ACIP measures).

If $m_{1}$ is an invariant probability measure on $\left(\mathbb{P}^{1}, \mathcal{B}, f\right)$ which is absolutely continuous with respect to Lebesgue measure, then we call $m_{1}$ an acip measure. If $f$ is a finite postcritical analytic map of $\mathbb{P}^{1}$, then there exists an acip measure for $f$ and, further, $f$ is exact with respect to this measure $([\mathrm{E}-\mathrm{L}])$. Corollary 4.3 implies that $m_{n, \pi}$ is an exact acip measure for $\left(\mathbb{P}^{n}, \mathcal{B}_{n}, F_{n}\right)$.

Example 4.13 (Type III measures).

Bruin and Hawkins $[\mathrm{Br}-\mathrm{H}]$ constructed measures $\nu$ on $\mathbb{P}^{1}$ for certain analytic maps which are ergodic, conservative, and exact, and admit no $\sigma$-finite invariant probability measure absolutely continuous with respect to $\nu$ (type III measures). We can use the type III measures on $\mathbb{P}^{1}$ constructed in $[\mathrm{Br}-\mathrm{H}]$ (an example of such a map and measure is explained in further detail in Example [5.5 of this paper) to construct type III measures on $\mathbb{P}^{2}$. Let $\nu$ be the ergodic type III measure for the rational map $R_{\wp, 2}$ constructed in $[\overline{\mathrm{Br}-\mathrm{H}}$. Let $m$ be the unique measure of maximal entropy for $R_{\wp, 2}$. Recall that $m$ lies in the smooth measure class and, by [B], $[\mathrm{B}-\mathrm{K}]$, is one-sided Bernoulli of entropy $\log 4$. Then by $[\mathrm{Br}-\mathrm{H}], \nu \times m$ is type III for $\hat{f}$ on $\mathbb{P}^{1} \times \mathbb{P}^{1}$.

Define a measure on $\mathbb{P}^{2}$ by $\lambda=\pi^{-1}(\nu \times m)$. The methods of [Br-H] can be used to show that $\lambda$ is type III for $F_{2}$ on $\mathbb{P}^{2}$. Namely, if there existed some invariant measure $\varrho$ absolutely continuous with respect to $\lambda$, then $\varrho$ would have to lift to an invariant measure on $\mathbb{P}^{1} \times \mathbb{P}^{1}$. This would contradict the fact that $\nu \times m$ is type III.

Example 4.14 (Ergodicity does not pass through the Ueda Construction).

Corollary 4.3 lists many measure-theoretic properties that pass through the Ueda construction. Here we note that there are examples of an ergodic measure $m_{1}$ on $\left(\mathbb{P}^{1}, \mathcal{B}, f\right)$ such that the resulting Ueda map $\left(\mathbb{P}^{2}, \mathcal{B}_{2}, m_{2, \pi}, F_{2}\right)$ is not ergodic. In fact, if we had an ergodic, not weak-mixing measure $m_{1}$ for $f$, then the P-D factor $\left(F_{2}, m_{2, \pi}\right)$ will not be ergodic. We construct such an ergodic but not weak-mixing measure $m_{1}$ for an analytic map $f$ of $\mathbb{P}^{1}$ in Example 5.15.

\section{Rational Maps of the Sphere with Parabolic Orbifold and Julia Set Equal to the Entire Sphere}

Recall that an analytic map of $\mathbb{P}^{1}$ can be viewed as a rational map $R$ of the complex Riemann sphere $\mathbb{C}_{\infty}$. In this section, we study the measure-theoretic properties of critically finite rational maps with parabolic orbifold and Julia set equal to the entire sphere. We define a measurable structure making these maps $k$-to-one finite factors of toral endomorphisms. We then use previous results of this paper to obtain the ergodic theoretic structure for a given nonsingular measure.

5.1. Parabolic Orbifolds. The notion of an orbifold was developed by Thurston [T]; here, we are only concerned with orbifolds that are homeomorphic to the sphere $S^{2}$. In this setting, an orbifold can be thought of as the sphere $S^{2}$, a finite collection of marked points $p_{1}, \ldots, p_{k} \in S^{2}$, and a function $v: S^{2} \rightarrow\{1,2,3, \ldots\} \cup$ 
$\infty$ where $v(x)=1$ if and only if $x \neq p_{j}$. Thus we can denote an orbifold by $\mathcal{O}=\left(S^{2}, v\left(p_{1}\right), \ldots, v\left(p_{k}\right)\right)$. We define the Euler characteristic of the orbifold $\mathcal{O}$ by

$$
\chi(\mathcal{O})=2-\sum_{j=1}^{k}\left(1-\frac{1}{v\left(p_{j}\right)}\right) .
$$

An orbifold is parabolic if $\chi(\mathcal{O})=0$. Using eq. (6), we see that there are exactly six parabolic orbifolds homeomorphic to $S^{2}$ :

$$
\begin{array}{ll}
\text { 1) } & \left(S^{2}, \infty, \infty\right), \\
\text { 2) } & \left(S^{2}, 2,2, \infty\right), \\
\text { 3) } & \left(S^{2}, 2,4,4\right), \\
\text { 4) } & \left(S^{2}, 2,3,6\right), \\
\text { 5) } & \left(S^{2}, 3,3,3\right), \\
6) & \left(S^{2}, 2,2,2,2\right) .
\end{array}
$$

Recall that the postcritical set $P(R)$ of a rational map $R$ of the Riemann sphere is defined by

$$
P(R)=\overline{\bigcup_{j>0}\left\{R^{j}(c): c \text { is a critical point of } R\right\}} .
$$

If there are a finite number of elements in $P(R)$, we can construct an orbifold corresponding to $R$ in the following way. Let $p_{1}, \ldots, p_{k}$ be the elements of $P(R)$. We define the function $v: S^{2} \rightarrow\{1,2,3, \ldots\} \cup \infty$ by

$$
v(z)=1 \text { if } z \text { is not in } P(R),
$$

$v(z)$ is the least common multiple of $v(y) \operatorname{deg}_{y}(R)$ for each $y \in R^{-1} z$,

where $\operatorname{deg}_{y}(R)$ is the local degree of $R$ at $y$. We note that if $c$ is a critical point such that $R(c)=c$, then $v(c)=\infty$.

Let $\operatorname{Im}(\tau)>0$ and let $\rho=\exp \left(\frac{i \pi}{3}\right)$. The six orbifolds listed in eq. (7) result from rational maps $R$ that are obtained from group endomorphisms of $\mathbb{C} / \Lambda$ where $\Lambda$ is given by the following groups (the numbers in this list correspond to the numbers in eq. (7)):

$$
\begin{aligned}
& \text { 1) } \Lambda=\langle u \mapsto u+1\rangle, \\
& \text { 2) } \Lambda=\langle u \mapsto u+1, u \mapsto-u\rangle, \\
& \text { 3) } \Lambda=\langle u \mapsto u+1, u \mapsto i u\rangle, \\
& \text { 4) } \Lambda=\langle u \mapsto u+1, u \mapsto \rho u\rangle, \\
& \text { 5) } \Lambda=\left\langle u \mapsto u+1, u \mapsto \rho^{2} u\right\rangle, \\
& \text { 6) } \Lambda=\langle u \mapsto u+1, u \mapsto u+\tau, u \mapsto-u\rangle .
\end{aligned}
$$

We refer the interested reader to $[\mathrm{E}-\mathrm{L}],[\mathrm{T}]$, or $[\mathrm{D}-\mathrm{H}]$ for more details. The orbifold $\left(S^{2}, \infty, \infty\right)$ corresponds to the rational maps of the form $R(z)=z^{d}$. The Tchebychev polynomials are the rational maps with orbifold $\left(S^{2}, 2,2, \infty\right)$. The rational maps with orbifolds listed in 3)-6) all have $J_{R}=\mathbb{C}_{\infty}$. We restrict our attention to cases 3$)-6$ ), as the corresponding rational maps of the sphere can be obtained as "meromorphic factors" of toral endomorphisms under the above identifications. We show this construction next. 
5.2. Elliptic Functions. Let $\alpha, \beta \in \mathbb{C}-\{0\}$ be such that $\alpha / \beta$ is not a real number. We define a lattice of points in the complex plane by $\Gamma=[\alpha, \beta]=\{\alpha k+\beta j$ : $k, j \in \mathbb{Z}\}$. Given a lattice $\Gamma, \mathbb{C} / \Gamma$ defines a complex torus.

Let $\Omega$ be defined by $\Omega=\{z \in \mathbb{C}: \operatorname{Im}(z)>0,-1 / 2 \leq \operatorname{Re}(z)<1 / 2,|z| \geq$ $1, \operatorname{Re}(z) \leq 0$ if $|z|=1\}$. There is a unique number $\tau$ in the region $\Omega$ such that the torus generated by $[1, \tau]$ is the same as the torus generated by $[\alpha, \beta]$ (see $[\overline{\mathrm{Be}} 2]$ ). For the rest of our discussion we use this representative $\tau$.

A meromorphic function which is periodic with respect to the lattice $\Gamma_{\tau}$ is said to be doubly periodic and is called an elliptic function. Hence we can view an elliptic function $Q_{\Gamma_{\tau}}$ as an analytic map $Q_{\Gamma_{\tau}}: \mathbb{C} / \Gamma_{\tau} \rightarrow \mathbb{C}_{\infty}$. For a rational map to belong to cases 3)-6) in eq. (7), the elliptic function $Q_{\Gamma_{\tau}}$ must be one of four functions. For simplicity in notation, we will abbreviate $Q_{\Gamma_{\tau}}$ by $Q$. When there is no confusion about which lattice we are working on, we denote $\Gamma_{\tau}$ by $\Gamma$.

5.2.1. The Weierstrass $\wp$ Function and $(2,2,2,2)$ Orbifolds. One well-studied elliptic function is the Weierstrass elliptic function, defined by

$$
\wp_{\Gamma}(u)=\frac{1}{u^{2}}+\sum_{v \in \Gamma-\{0\}}\left[\frac{1}{(u-v)^{2}}-\frac{1}{v^{2}}\right] .
$$

From the definition, we see that $\wp_{\Gamma}(u)=\wp_{\Gamma}(-u)$ and thus $\wp_{\Gamma}$ is an even function. When the lattice $\Gamma$ is understood, we abbreviate $\wp_{\Gamma}$ by $\wp$. Using the Weierstrass elliptic function, we obtain the complex sphere $\mathbb{C}_{\infty}$ as the quotient of $\mathbb{C}$ by $u \rightarrow$ $u+1, u \rightarrow u+\tau$, and $u \rightarrow-u$. Hence we are in case 6 ) of eq. (8).

From the definition of $\wp$ we have the following homogeneity property for $\lambda \in$ $\mathbb{C}-\{0\}$.

$$
\wp_{\lambda \Gamma}(\lambda u)=\frac{1}{\lambda^{2}} \wp_{\Gamma}(u) .
$$

5.2.2. The Elliptic Function $\wp^{2}$ and $(2,4,4)$ Orbifolds. Here we work with the lattice $\Gamma_{i}=[1, i]$. The lattice $\Gamma_{i}$ has the property that $i \Gamma_{i}=\Gamma_{i}$. Using eq. (9), the Weierstrass elliptic function on $\Gamma_{i}$ satisfies the following equation:

$$
\wp_{\Gamma_{i}}(i u)=\wp_{i \Gamma_{i}}(i u)=\frac{1}{i^{2}} \wp_{\Gamma_{i}}(u)=-\wp_{\Gamma_{i}}(u) .
$$

We emphasize that, while the identity for the Weierstrass $\wp$-function $\wp(z)=\wp(-z)$ holds on any lattice $\Gamma_{\tau}$, eq. (10) holds only on the lattice $\Gamma_{i}$.

Squaring both sides of eq. (10), we see that $\wp^{2}(i u)=\wp^{2}(u)$. Since $\wp$ is an even function, so is $\wp^{2}$. Hence we have the following equalities:

$$
\wp^{2}(i u)=\wp^{2}(u)=\wp^{2}(-u)=\wp^{2}(-i u) .
$$

Further, $\wp^{2}$ is also an elliptic function (see Lang $[\mathrm{L}]$ for a proof). Thus we see that the elliptic function $\wp^{2}$ identifies $u$ with $i u$ and we are in case 3 ) of eq. (8).

5.2.3. The Elliptic Function $\wp^{\prime}$ and $(3,3,3)$ Orbifolds. The derivative $\wp^{\prime}$ of the Weierstrass elliptic function satisfies the following homogeneity property (see $[\mathrm{L}]$ ).

$$
\wp_{\lambda \Gamma}^{\prime}(\lambda u)=\frac{1}{\lambda^{3}} \wp_{\Gamma}^{\prime}(u)
$$

for any $\lambda \in \mathbb{C}-\{0\}$. Let $\rho=\frac{1+i \sqrt{3}}{2}$ and let $\Gamma_{\rho^{2}}=\left[1, \rho^{2}\right]$. We see that $\Gamma_{\rho^{2}}$ has the property that $\rho \Gamma_{\rho^{2}}=\Gamma_{\rho^{2}}$. In particular, this implies that $\rho^{j} \Gamma_{\rho^{2}}=\Gamma_{\rho^{2}}$ for 
$j=1, \ldots, 6$. Using eq. (11), we have

$$
\begin{aligned}
& \wp_{\Gamma_{\rho^{2}}}^{\prime}\left(\rho^{2} u\right)=\wp_{\rho^{2} \Gamma_{\rho^{2}}}^{\prime}\left(\rho^{2} u\right)=\frac{1}{\rho^{6}} \wp_{\Gamma_{\rho^{2}}}^{\prime}(u)=\wp_{\Gamma_{\rho^{2}}}^{\prime}(u), \\
& \wp_{\Gamma_{\rho^{2}}}^{\prime}\left(\rho^{4} u\right)=\wp_{\rho^{4} \Gamma_{\rho^{2}}}^{\prime}\left(\rho^{4} u\right)=\frac{1}{\rho^{12}} \wp_{\Gamma_{\rho^{2}}}^{\prime}(u)=\wp_{\Gamma_{\rho^{2}}}^{\prime}(u) .
\end{aligned}
$$

Thus we have the following equalities:

$$
\wp^{\prime}(u)=\wp^{\prime}\left(\rho^{2} u\right)=\wp^{\prime}\left(\rho^{4} u\right) .
$$

The identification $u \rightarrow \rho^{2} u$ implies that we are in case 5 ) of eq. (8).

5.2.4. The Elliptic Function $\left(\wp^{\prime}\right)^{2}$ and $(2,3,6)$ Orbifolds. Again, we use $\rho=\frac{1+i \sqrt{3}}{2}$ and the lattice $\Gamma_{\rho^{2}}=\left[1, \rho^{2}\right]$. Using eq. (11), we obtain the following:

$$
\wp_{\Gamma_{\rho^{2}}}^{\prime}\left(\rho^{j} u\right)=\wp_{\rho^{j}}^{\prime} \Gamma_{\rho^{2}}\left(\rho^{j} u\right)=\frac{1}{\rho^{3} j} \wp_{\Gamma_{\rho^{2}}}^{\prime}(u)=-\wp_{\Gamma_{\rho^{2}}}^{\prime}(u) \text { for } j=1,3,5 .
$$

Squaring both sides of eq. (13) and using eq. (12), we obtain

$$
\wp^{\prime 2}(u)=\wp^{\prime 2}(\rho u)=\wp^{\prime 2}\left(\rho^{2} u\right)=\wp^{\prime 2}\left(\rho^{3} u\right)=\wp^{\prime 2}\left(\rho^{4} u\right)=\wp^{\prime 2}\left(\rho^{5} u\right) .
$$

As $\left(\wp^{\prime}\right)^{2}$ identifies $u \rightarrow \rho u$, we are in case 4$)$ of eq. (8).

5.3. Construction of the Dynamical System. We use the elliptic functions $\wp, \wp^{\prime}, \wp^{2}$, and $\left(\wp^{\prime}\right)^{2}$ to construct rational maps of the complex sphere $\mathbb{C}_{\infty}$ which have the orbifolds listed in eq. (7). We are interested in the toral endomorphisms which give a well-defined rational map with respect to semi-conjugation by one of the four elliptic functions. Specifically, we want toral endomorphisms $T: \mathbb{C} / \Gamma_{\tau} \rightarrow \mathbb{C} / \Gamma_{\tau}$ of the form $T(u)=\omega u+\kappa$ (where $\omega$ is an algebraic integer in the imaginary quadratic extension $\mathbb{Q}(\tau)$, and $\kappa$ is a complex number), such that $T$ satisfies the following (see $[\mathrm{Mi}],[\mathrm{D}-\mathrm{H}])$ :

1) If $Q=\wp$, then $T(-u)=-T(u)$.

2) If $Q=\wp^{2}$, then $T(i u)=i T(u)$.

3) If $Q=\wp^{\prime}$, then $T\left(\rho^{2} u\right)=\rho^{2} T(u)$.

4) If $Q=\left(\wp^{\prime}\right)^{2}$, then $T(\rho u)=\rho T(u)$.

For example, if $\omega \Gamma \subset \Gamma$ and $2 \kappa \in \Gamma$, then $T(u)=\omega u+\kappa$ satisfies eq. (14)(1) and thus $\wp \circ T \circ \wp^{-1}$ is well-defined.

Let $Q \in\left\{\wp^{2},\left(\wp^{\prime}\right)^{2}, \wp^{\prime}, \wp\right\}$, and let $T: \mathbb{C} / \Gamma \rightarrow \mathbb{C} / \Gamma$ defined by $T(u)=\omega u+\kappa$ be in the list of acceptable endomorphisms for $Q$ given in eq. (14). We define a $\operatorname{map} R_{Q_{\Gamma}, T}=R_{Q, T}: \mathbb{C}_{\infty} \rightarrow \mathbb{C}_{\infty}$ by $R_{Q, T}(z)=Q \circ T \circ Q^{-1}(z)$ as in the following diagram:

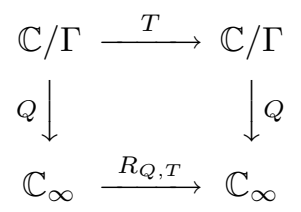

Since $R_{Q, T}$ is locally a composition of meromorphic maps, $R_{Q, T}$ itself must be analytic and hence rational. We note that the degree of $T$ and thus $R_{Q, T}$ is $\omega \bar{\omega}=d$. 
Example 5.1. We use the lattice $\Gamma=[1, i \sqrt{2}]$, the endomorphism $T(u)=i \sqrt{2} u$, and the elliptic function $\wp$. The map $R_{\wp, i \sqrt{2}}(z)=\wp \circ T \circ \wp^{-1}(z)$ is a degree two rational map of the sphere. We note that McMullen [Mc1] showed that

$$
R_{\wp, i \sqrt{2}}(z)=\frac{z^{2}+1+\sqrt{2}}{z^{2}-1-\sqrt{2}} .
$$

5.4. Weierstrass Meromorphic Factor Maps. Let $\mathcal{C}_{1}$ be the $\sigma$-algebra of Borel sets on $\mathbb{C} / \Gamma$ and $\mu$ be a nonsingular measure on $\mathcal{C}_{1}$. Let $\mathcal{C}_{2}$ be the $\sigma$-algebra of Borel sets on $\mathbb{C}_{\infty}$ and define the measure $\nu_{Q}$ by $\nu_{Q}(E)=\mu\left(Q^{-1} E\right)$ for all $E \in \mathcal{C}_{2}$. We call a dynamical system obtained in the above manner a Weierstrass meromorphic factor of the toral endomorphism.

The following lemma will simplify some proofs later in this paper.

Lemma 5.2. For any $T$ satisfying eq. (14) $(3)$ and $(4),\left(\mathbb{C}_{\infty}, \mathcal{C}_{2}, \nu_{\left(\wp^{\prime}\right)^{2}}, R_{\left(\wp^{\prime}\right)^{2}, T}\right)$ is a two-to-one factor of $\left(\mathbb{C}_{\infty}, \mathcal{C}_{2}, \nu_{\wp^{\prime}}, R_{\wp^{\prime}, T}\right)$.

Proof. The factor map is given by $\theta(z)=z^{2}$ and we have $\theta R_{\wp^{\prime}, T}=R_{\left(\wp^{\prime}\right)^{2}, T} \theta$ for all $z \in \mathbb{C}_{\infty}$. Further, by the definition of the measures $\nu_{\wp^{\prime}}$ and $\nu_{\left(\wp^{\prime}\right)^{2}}$, we have $\nu_{\left(\wp^{\prime}\right)^{2}}=\nu_{\wp^{\prime}} \circ \theta^{-1}$.

The following corollary follows from the results of Section 3

Corollary 5.3. Let $\mu$ be any nonatomic measure on $\mathbb{C} / \Gamma$. Suppose $\left(\mathbb{C} / \Gamma, \mathcal{C}_{1}, \mu, T\right)$ has any of the following properties and let $R_{Q, T}$ be a Weierstrass meromorphic factor of $\left(\mathbb{C} / \Gamma, \mathcal{C}_{1}, \mu, T\right)$ :

1. nonsingular,

2. measure-preserving,

3. weak-mixing,

4. strong-mixing,

5. exact,

6. automorphically Bernoulli.

Then $\left(\mathbb{C}_{\infty}, \mathcal{C}_{2}, \nu_{Q}, R_{Q, T}\right)$ has the same property.

Proof. The result follows from Lemma 3.7

Theorem 5.4. Let $\mu$ be any nonatomic, invariant Borel measure on $\mathbb{C} / \Gamma$. Let $R_{Q, T}$ be a Weierstrass meromorphic factor of $\left(\mathbb{C} / \Gamma, \mathcal{C}_{1}, \mu, T\right)$. Then $h_{\mu}(T)=$ $h_{\nu}\left(R_{Q, T}\right)$.

Proof. Fix an endomorphism $T$ of the torus $\left(\mathbb{C} / \Gamma, \mathcal{C}_{1}, \mu\right)$. We claim that $\left(\mathbb{C}_{\infty}, \mathcal{C}_{2}, \nu_{Q}\right.$, $\left.R_{Q, T}\right)$ is a finite factor of $\left(\mathbb{C} / \Gamma, \mathcal{C}_{1}, \mu, T\right)$. Thus we can use Proposition 3.4 to complete the proof.

We have previously shown that $\left(\mathbb{C}_{\infty}, \mathcal{C}_{2}, \nu_{Q}, R_{Q, T}\right)$ is a measure-theoretic factor of $\left(\mathbb{C} / \Gamma, \mathcal{C}_{1}, \mu, T\right)$. To prove the claim, we first need to find a partition of the torus into $k$ sets such that $Q$ is one-to-one on each set. We represent the torus $\mathbb{C} / \Gamma$ by a period parallelogram $U_{\tau}=\{c+d \tau \in \mathbb{C}: 0 \leq c, d<1\}$, and we rewrite the map $T$ of $\mathbb{C} / \Gamma$ as a map of $U_{\tau}$.

First, when $Q=\wp$ we can use any torus $\Gamma_{\tau}$. We illustrate the partition of $U$ into 2 pieces $\gamma_{\wp}=\left\{\gamma_{1}, \gamma_{2}\right\}$ in Figure 6. We see that $\gamma_{2}=-\gamma_{1}$. This implies that $\wp$ is one-to-one on each $\gamma_{j}$ and $\left(\mathbb{C}_{\infty}, \mathcal{C}_{2}, \nu_{\wp}, R_{\wp, T}\right)$ is a finite factor of $\left(U_{\tau}, \mathcal{C}_{1}, \mu, T\right)$. 


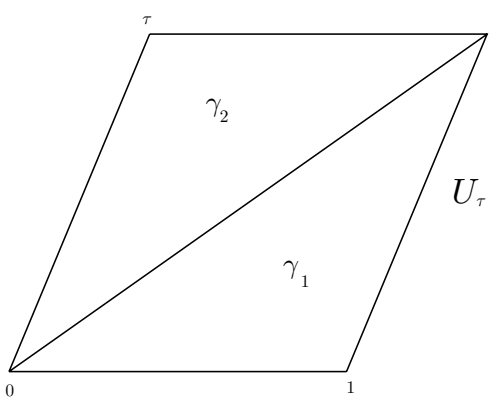

FiguRE 6. $\gamma_{\wp}$

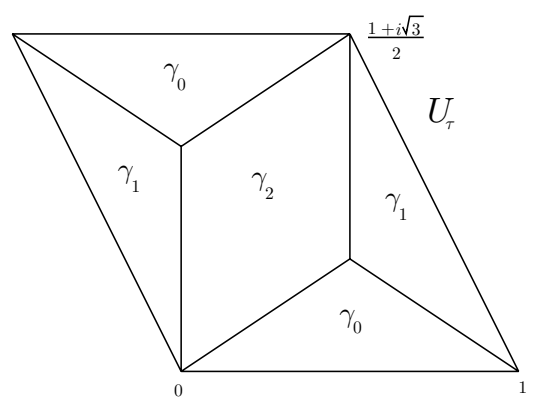

FIGURE 8. $\gamma_{\wp^{\prime}}$

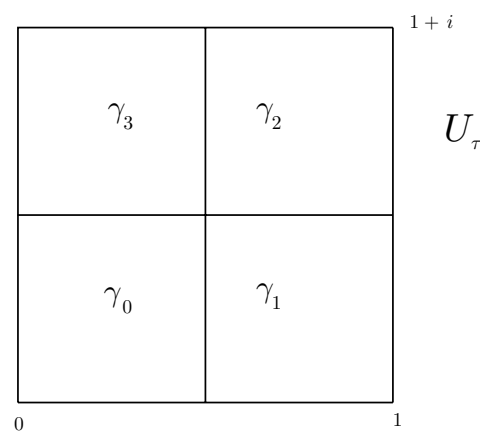

FIGURE 7. $\gamma_{\wp 2}$

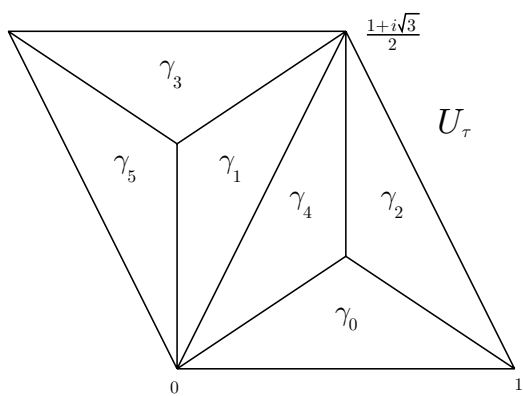

FIGURE $9 . \gamma_{\wp^{\prime 2}}$

Recall that if $Q=\wp^{2}$, then the homogeneity property of $\wp^{2}$ implies that we must use the lattice $\Gamma_{i}=[1, i]([\mathrm{L}])$. We partition $U_{i}$ into 4 pieces by $\gamma_{\wp^{2}}=\left\{\gamma_{0}, \gamma_{1}, \gamma_{2}, \gamma_{3}\right\}$ where

$$
\begin{gathered}
\gamma_{j}=\left\{c+d i \in U: \frac{j}{2} \leq c<\frac{j+1}{2}, 0 \leq d<\frac{1}{2}, j=0,1\right\} \\
\gamma_{j}=\left\{c+d i \in U: \frac{(j-1) \bmod 2}{2} \leq c<\frac{[(j-1) \bmod 2]+1}{2}, \frac{1}{2} \leq d<1, j=2,3\right\}
\end{gathered}
$$

(see Figure 7). We see that $i \gamma_{j}=\gamma_{k}$ where $k=j+1(\bmod 4)$. Thus $\wp^{2}$ is one-toone on each set $\gamma_{j}$ and we have shown that $\left(\mathbb{C}_{\infty}, \mathcal{C}_{2}, \nu_{\wp}, R_{\wp^{2}, T}\right)$ is a finite factor of $\left(U_{i}, \mathcal{C}_{1}, \mu, T\right)$.

If $Q=\wp^{\prime}$, then the homogeneity property of $\wp^{\prime}$ (see [L]) implies that we use the lattice $\Gamma_{\rho^{2}}$, where $\rho=\frac{1+i \sqrt{3}}{2}$. We illustrate $\gamma_{\wp^{\prime}}=\left\{\gamma_{0}, \gamma_{1}, \gamma_{2}\right\}$ in Figure 8. We see that $\rho^{2} \gamma_{j}=\gamma_{k}$ where $k=j+1(\bmod 3)$. Again, this implies that $\wp^{\prime}$ is one-to-one on each $\gamma_{j}$ and $\left(\mathbb{C}_{\infty}, \mathcal{C}_{2}, \nu_{\wp^{\prime}}, R_{\wp^{\prime}, T}\right)$ is a finite factor of $\left(U_{\rho^{2}}, \mathcal{C}_{1}, \mu, T\right)$.

Finally, when $Q=\wp^{\prime 2}$, we also use the lattice $\Gamma_{\rho^{2}}$. We illustrate $\gamma_{\wp^{\prime}}=$ $\left\{\gamma_{0}, \gamma_{1}, \gamma_{2}, \ldots, \gamma_{5}\right\}$ in Figure 9. We see that $\rho \gamma_{j}=\gamma_{k}$ where $k=j+1(\bmod 6)$. Thus $\wp^{\prime 2}$ is one-to-one on each $\gamma_{j}$ and $\left(\mathbb{C}_{\infty}, \mathcal{C}_{2}, \nu_{\wp^{\prime 2}}, R_{\wp^{\prime}}{ }^{2}, T\right)$ is a finite factor of $\left(U_{\rho^{2}}, \mathcal{C}_{1}, \mu, T\right)$. 
With respect to each elliptic function $\wp, \wp^{\prime}, \wp^{2}$ and $\left(\wp^{\prime}\right)^{2}$ we have shown that $\left(\mathbb{C}_{\infty}, \mathcal{C}_{2}, \nu_{Q}, R_{Q, T}\right)$ is a finite factor of $\left(\mathbb{C} / \Gamma, \mathcal{C}_{1}, \mu, T_{\omega}\right)$. The result then follows from Proposition 3.4

Example 5.5 (Type III measures).

Bruin and Hawkins $\mathrm{Br}-\mathrm{H}]$ constructed measures $\nu$ on the sphere for rational maps of the form $R_{\wp, T}$ with $T(u)=n u(n=2,3,4, \ldots)$ which are ergodic, conservative, and exact, and admit no $\sigma$-finite invariant probability measure absolutely continuous with respect to $\nu$ (type III measures). Their methods can be extended to rational maps of the sphere of the form $R_{Q, T}(n=2,3,4, \ldots)$ with $Q \in\left\{\wp^{2}, \wp^{\prime},\left(\wp^{\prime}\right)^{2}\right\}$.

Bruin and Hawkins begin with an ergodic type III measure $\rho$ on the interval and normalized Lebesgue measure $m$ on the interval and show that the measure $\mu$ defined by $\mu=\rho \times m$ is a type III measure on the torus. They prove that the measure $\nu=\wp^{-1} \circ \mu$ is a type III measure on the sphere by arguing that if there existed some other invariant measure $\rho$ absolutely continuous with respect to $\mu$, then $\rho$ would lift to an invariant measure on the torus absolutely continuous with respect to $\mu$. This would contradict the fact that $\mu$ was type III, and hence $\nu$ is type III. Bruin and Hawkins' argument easily extends to rational maps which are formed by semi-conjugating toral endomorphisms of the form $T(u)=n u$ by the elliptic functions $\wp^{2}, \wp^{\prime}$ and $\left(\wp^{\prime}\right)^{2}$.

5.5. Topological Pressure and Equilibrium States. Lyubich Ly2 proved that an equilibrium state exists for any rational map $R$ and any continuous function $g: \mathbb{C}_{\infty} \rightarrow \mathbb{R}$. In $[\mathrm{D}-\mathrm{U}]$, Denker and Urbanski give a technical condition guaranteeing the uniqueness of an equilibrium state. Here, we prove how the pressure on the sphere is related to pressure on the torus for critically finite rational maps with parabolic orbifold and $J_{R}=\mathbb{C}_{\infty}$. Theorem 5.9 is a strengthening of Denker and Urbanski's result for these maps.

Theorem 5.6. Let $T: \mathbb{C} / \Gamma \rightarrow \mathbb{C} / \Gamma$ be a complex toral endomorphism and let $g: \mathbb{C}_{\infty} \rightarrow \mathbb{R}$ be a continuous function. Then $P\left(R_{Q, T}, g\right)=P\left(T, \frac{g \circ Q}{\operatorname{deg} Q}\right)$ where $\operatorname{deg} Q$ (the degree of $Q$ ) is $2,3,4$, or 6 when $Q=\wp, \wp^{\prime}, \wp^{2}$, or $\left(\wp^{\prime}\right)^{2}$ respectively.

Proof. The map $\frac{g \circ Q}{\operatorname{deg} Q}: \mathbb{C} / \Gamma \rightarrow \mathbb{R}$ is the composition of two continuous functions and is itself continuous. We know that any $T$-invariant measure $\mu$ on $\mathbb{C} / \Gamma$ determines a $R_{Q, T}$-invariant measure $\nu_{Q}$ on $\mathbb{C}_{\infty}$. Further, any $R_{Q, T}$-invariant measure on $\mathbb{C}_{\infty}$ arises from a $T$-invariant measure on $\mathbb{C} / \Gamma$ in the following manner. Define $\gamma_{Q}$ as in the proof of Theorem [5.4 If $\lambda$ is a $R_{Q, T}$-invariant measure on $\mathbb{C}_{\infty}$, then $\lambda_{*}$ on $\mathbb{C} / \Gamma$ defined by

$$
\lambda_{*}(B)=\sum_{j=1}^{\operatorname{deg} Q} \frac{\lambda\left(Q\left(B \cap \gamma_{j}\right)\right)}{\operatorname{deg} Q}
$$

is invariant. Thus any invariant measure on $\mathbb{C}_{\infty}$ is associated with an invariant measure on $\mathbb{C} / \Gamma$, and we will denote these related measures by $\mu$ on $\mathbb{C} / \Gamma$ and $\nu_{Q}$ on $\mathbb{C}_{\infty}$. Theorem 5.4 implies that $h_{\mu}(T)=h_{\nu_{Q}}\left(R_{Q, T}\right)$. 
Let $\mu$ be a $T$-invariant measure on $\mathbb{C} / \Gamma$. We have

$$
\begin{aligned}
\int_{\mathbb{C} / \Gamma} \frac{g \circ Q}{\operatorname{deg} Q} d \mu & =\sum_{j=1}^{\operatorname{deg} Q} \int_{\gamma_{j}} \frac{g \circ Q}{\operatorname{deg} Q} d \mu=\operatorname{deg} Q \int_{\gamma_{1}} \frac{g \circ Q}{\operatorname{deg} Q} d \mu \\
& =\int_{\gamma_{1}} g \circ Q d \mu=\int_{\mathbb{C}_{\infty}} g d \nu_{Q} .
\end{aligned}
$$

Thus

$$
\begin{gathered}
P\left(R_{Q, T}, g\right)=\sup \left\{h_{\nu_{Q}}\left(R_{Q, T}\right)+\int g d \nu_{Q}\right\}=\sup \left\{h_{\mu}(T)+\int \frac{g \circ Q}{\operatorname{deg} Q} d \mu\right\} \\
=P\left(T, \frac{g \circ Q}{\operatorname{deg} Q}\right) .
\end{gathered}
$$

If $(X, r)$ is a metric space, we say that a continuous function $g: X \rightarrow \mathbb{R}$ is Hölder continuous if there are constants $\alpha$ and $\beta, 0 \leq \beta \leq 1$, such that $|g(x)-g(y)| \leq$ $\alpha r(x, y)^{\beta}$ for all $x, y \in X$. Denker and Urbanski [D-U] proved the following theorem guaranteeing when a continuous map $g: \mathbb{C}_{\infty} \rightarrow \mathbb{R}$ will have a unique equilibrium state.

Theorem 5.7 ([D-U]). Let $R$ be a rational map of the sphere and $g: \mathbb{C}_{\infty} \rightarrow \mathbb{R}$ be a Hölder continuous function such that $P(R, g)>\sup _{z \in \mathbb{C}_{\infty}} g(z)$. Then there exists exactly one equilibrium state $\nu_{g}$ for $g$ and $\left(\mathbb{C}_{\infty}, \mathcal{B}, \nu_{g}, R\right)$ is exact.

We can obtain a stronger result for critically finite rational maps of the sphere with parabolic orbifold and $J_{R}=\mathbb{C}_{\infty}$ since these maps are factors of expanding toral endomorphisms. Walters [W2] proved the following theorem for expanding maps, extending results of $[\mathrm{Bo}],[\mathrm{Ke}],[\mathrm{Ru}]$.

Theorem 5.8 ([W2]). Let $L: X \rightarrow X$ be a $C^{1}$ expanding map and let $g: X \rightarrow \mathbb{R}$ be a Hölder continuous function. Then $g$ has a unique equilibrium state $\mu_{g}$. Further, $\mu_{g}$ is nonatomic and positive on nonempty open sets. Also, $\left(X, \mu_{g}, L\right)$ is exact and automorphically Bernoulli.

Of course, rational maps of the sphere of the form $R_{Q, T}$ are not expanding since $J_{R_{Q, T}}=\mathbb{C}_{\infty}$ and hence the Julia set contains critical points. However, we can use Walters' theorem on the torus and the theorems of this chapter to obtain the following theorem.

Theorem 5.9. Let $R_{Q, T}$ be a critically finite rational map with parabolic orbifold and $J_{R_{Q, T}}=\mathbb{C}_{\infty}$, and let $g: \mathbb{C}_{\infty} \rightarrow \mathbb{R}$ be a continuous function with $g \circ Q: \mathbb{C} / \Gamma \rightarrow \mathbb{R}$ Hölder continuous. Then $g$ has a unique equilibrium state $\nu_{g}$ and $\left(\mathbb{C}_{\infty}, \mathcal{B}, \nu_{g}, R_{Q, T}\right)$ is exact and automorphically Bernoulli.

Proof. The result follows from Theorems [5.6] and 5.8 and Corollary [5.3]

5.6. One-sided Bernoulli Rational Maps. Next, we turn our attention to the unique measure of maximal entropy. Freire, Lopes, and Mañé [F-L-M] and Lyubich Ly2 conjectured that any rational map with its unique measure of maximal entropy is isomorphic to a one-sided Bernoulli shift.

The critically finite rational maps with parabolic orbifold and $J_{R} \neq \mathbb{C}_{\infty}$ are known to be one-sided Bernoulli. These maps are either of the form $R(z)=z^{d}$ (and have $J_{R}=S^{1}$ ) or are the Tchebychev polynomials (and have $J_{R}=[-2,2]$ ). 
Both are one-sided Bernoulli with respect to their maximal entropy measure, onedimensional Lebesgue measure restricted to $J_{R}$. In $[\mathrm{B}$ and $\mathrm{B}-\mathrm{K}$ ] rational maps of the form $R_{\wp, T}$ where $T(u)=n u$ (the integer Lattès maps) or $T(u)=(n+n i) u$ are proved to be one-sided Bernoulli with respect to a smooth measure.

In this section we use normalized two-dimensional Lebesgue measure on the period parallelogram $U_{\tau}$. To avoid confusion, we will use the notation leb to denote this measure. As before, we use the $\sigma$-algebra $\mathcal{C}_{2}$ of Borel sets on $\mathbb{C}_{\infty}$ and the measure $\nu_{Q}$ given by $\nu_{Q}(F)=\operatorname{leb}\left(Q^{-1} F\right)$ for all $F \in \mathcal{C}_{2}$. Since $T$ is measurepreserving with respect to leb ([W1]), Corollary 5.3 implies that $R_{Q, T}$ preserves $\nu_{Q}$. Further, $\nu_{Q}$ is equivalent to the normalized surface area measure on $\mathbb{C}_{\infty}$.

Some, but not all, toral endomorphisms are known to be one-sided Bernoulli with respect to a smooth measure on the torus. We state the results of Ito II and Ito and Ohtsuki [I-O for toral endomorphisms.

Theorem $5.10([\mathrm{I}],[\mathrm{I}-\mathrm{O}])$. Let $T(u)=\omega u$ be one of the following complex toral endomorphisms, and let leb be normalized Lebesgue measure on $U_{\tau}$ :

1. $\omega=a+i b, a, b \in \mathbb{Z}$,

2. $\omega=-n \pm i \sqrt{q},(n=1,2,3, \ldots),-q=2,3(\bmod 4)$,

3. $\omega=\frac{-2 n+1 \pm i \sqrt{q}}{2},(n=1,2,3, \ldots),-q=1(\bmod 4)$,

4. $\omega=\frac{-2 n+1 \pm i \sqrt{q}}{2},(n=2,3, \ldots),-q=3$.

Then $\left(\mathbb{C} / \Gamma, \mathcal{C}_{1}\right.$, leb, $\left.T\right)$ is isomorphic to a one-sided Bernoulli shift on $d=\operatorname{deg}\left(T_{\omega}\right)$ symbols with measure $p_{d}$ given by $\vec{p}=(1 / d, \ldots, 1 / d)$.

However, even in the cases when $\left(\mathbb{C} / \Gamma, \mathcal{C}_{1}\right.$, leb, $\left.T_{\omega}\right)$ is isomorphic to a one-sided Bernoulli shift, it does not automatically follow that $\left(\mathbb{C}_{\infty}, \mathcal{C}_{2}, \nu_{Q}, R_{Q, T}\right)$ is one-sided Bernoulli, even though Corollary 5.3 and Theorem 5.4 imply that $\left(\mathbb{C}_{\infty}, \mathcal{C}_{2}, \nu_{Q}, R_{Q, T}\right)$ is exact, automorphically Bernoulli, and of maximal entropy.

Our main theorem of this section is as follows:

Theorem 5.11. With respect to each of the following elliptic functions $Q_{\Gamma_{\tau}}$ and degree $d$ toral endomorphisms $T$, the rational map $R_{Q, T}(z)=Q \circ T \circ Q^{-1}(z)$ with measure $\nu_{Q}$ on $\mathbb{C}_{\infty}$ is isomorphic to the one-sided $\left(\frac{1}{d}, \ldots, \frac{1}{d}\right)$ Bernoulli shift on $d$ symbols; i.e. $\left(\mathbb{C}_{\infty}, \mathcal{C}_{2}, \nu_{Q}, R_{Q, T}\right) \cong\left(X_{d}{ }^{+}, \mathcal{D}, p_{d}, \sigma\right)$ :

1. $Q=\wp, \quad \Gamma=[1, c i \sqrt{q}], T(u)=c i \sqrt{q} u, d=c^{2} q \in \mathbb{R}^{+}-\{0\}$,

2. $Q=\wp, \Gamma=\left[1, e^{\frac{\pi i}{3}}\right], T(u)=(n+n i \sqrt{3}) u$, where $n \in \mathbb{Z}-\{0\}, d=4 n^{2}$,

3. $Q=\wp, \Gamma=[1, i], T(u)=(n+l i) u$, where $n, l \in \mathbb{Z}-\{0\}$, exactly one of $n$, $l$ odd, $d=n^{2}+l^{2}$,

4. $Q=\wp^{2}, \Gamma=[1, i], T(u)=n u$, where $n \in \mathbb{Z}-\{0,1,-1\}, d=n^{2}$,

5. $Q=\wp^{2}, \Gamma=[1, i], \quad T(u)=$ niu, where $n \in \mathbb{Z}-\{0,1,-1\}, d=n^{2}$,

6. $Q=\wp^{2}, \Gamma=[1, i], T(u)=(n+n i) u$, where $n \in \mathbb{Z}-\{0\}, d=2 n^{2}$,

7. $Q=\wp^{2}, \quad \Gamma=[1, i], T(u)=(1+2 i) u, d=5$,

8. $Q=\left(\wp^{\prime}\right)^{2}, \Gamma=\left[1, e^{\frac{2 \pi i}{3}}\right], \quad T(u)=i \sqrt{3} u, d=3$,

9. $Q=\left(\wp^{\prime}\right)^{2}, \quad \Gamma=\left[1, e^{\frac{2 \pi i}{3}}\right], \quad T(u)=(1+i \sqrt{3}) u, d=4$,

10. $Q=\left(\wp^{\prime}\right)^{2}, \Gamma=\left[1, e^{\frac{2 \pi i}{3}}\right], \quad T(u)=2^{n} u$, where $n \in \mathbb{Z}-\{0\}, d=2^{2 n}$,

11. $Q=\left(\wp^{\prime}\right)^{2}, \Gamma=\left[1, e^{\frac{2 \pi i}{3}}\right], \quad T(u)=3^{n} u$, where $n \in \mathbb{Z}-\{0\}, d=3^{2 n}$,

12. $Q=\left(\wp^{\prime}\right), \Gamma=\left[1, e^{\frac{2 \pi i}{3}}\right], \quad T(u)=2^{n} u$, where $n \in \mathbb{Z}-\{0\}, d=2^{2 n}$,

13. $Q=\left(\wp^{\prime}\right), \quad \Gamma=\left[1, e^{\frac{2 \pi i}{3}}\right], \quad T(u)=3^{n} u$, where $n \in \mathbb{Z}-\{0\}, d=3^{2 n}$, 
14. $Q=\left(\wp^{\prime}\right), \quad \Gamma=\left[1, e^{\frac{2 \pi i}{3}}\right], \quad T(u)=i \sqrt{3} u, d=3$,

15. $Q=\left(\wp^{\prime}\right), \quad \Gamma=\left[1, e^{\frac{2 \pi i}{3}}\right], \quad T(u)=(1+i \sqrt{3}) u, d=4$.

By considering degrees or the behavior of the postcritical sets, we obtain nonconjugate rational maps in the following cases of Theorem 5.11 (1), (2), (3), (4), (6), (7), (10), (11). We first discuss some corollaries, and then prove Theorem 5.11 in Section 5.7 .

Corollary 5.12. For any $d>1$, there exists a rational map of the sphere which, with respect to a smooth invariant measure, is isomorphic to a one-sided Bernoulli shift of entropy $\log d$.

Proof. If $d>1$, then $d$ can be written uniquely as $d=c^{2} q$ where $q$ has no square factors. Then the corollary follows immediately from Theorem 5.11 (1).

Corollary 5.13. The toral endomorphisms listed in Theorem 5.11 are isomorphic to one-sided Bernoulli shifts.

Proof. The toral endomorphisms are two, three, four, or six point extensions of one-sided Bernoulli shifts. Hence by $[\mathrm{A}-\mathrm{G}-\mathrm{W}], \mathrm{P}$ they are one-sided Bernoulli.

We note that Corollary 5.13 gives a new proof of some results of Ito [I] and Ito-Ohtsuki [-O] (stated as Theorem 5.10 in this paper). Further, Corollary 5.13 provides us with new examples of toral endomorphisms which were not previously known to be one-sided Bernoulli of maximal entropy with respect to a smooth measure. For example, we now know that the toral endomorphism $T(u)=1+i \sqrt{3}$ is one-sided Bernoulli of entropy $\log 4$.

Corollary 5.14. If $F_{2}$ is a Ueda map constructed from any of the rational maps of the sphere in Theorem 5.11, then $F_{2}$ is isomorphic to a one-sided Bernoulli shift of maximal entropy with respect to a smooth invariant measure.

Proof. The proof follows from Theorem 5.11 Corollary 4.3, and Theorem 4.9.

Example 5.15 (An ergodic, not weak-mixing measure on the sphere).

We can use Theorem 5.11 to define an invariant, ergodic, not weak-mixing measure for a rational map of the sphere. We use the measure $\mu$ defined on $\left(X_{d}^{+}, \mathcal{C}, \sigma\right)$ by Eigen and Hawkins $[\mathrm{E}-\mathrm{H}]$ and discussed in Proposition 3.11 of this paper. Recall that $\mu$ was ergodic but not weak-mixing.

We begin with the rational map $R_{\wp, i \sqrt{2}}$ which is isomorphic to the two-shift by Theorem 5.11 In Section 5.7 we construct a point map $\phi$ between $\mathbb{C}_{\infty}$ and $X_{2}{ }^{+}$. Then $\nu=\phi^{-1} \circ \mu$ defines an ergodic, not weak-mixing measure for the rational map of the sphere $R_{\wp, i \sqrt{2}}$.

5.7. Proof of Theorem 5.11. To prove Theorem 5.11, we construct a direct isomorphism between the dynamical system and a one-sided Bernoulli shift using the fact that these maps are factors of toral endomorphisms. For a given rational map of the sphere, we find a measure-theoretic isomorphism between the rational map and the toral map restricted to a subset of its original domain using the measure induced by the factor map. We then find an independent generating partition of this space which gives an isomorphism to a one-sided Bernoulli shift of entropy $\log (\operatorname{deg} R)$. We change our notation slightly from the previous section to emphasize which toral endomorphism we are considering. All toral endomorphisms in this 
section are of the form $T_{\omega}(u)=\omega u$, and we denote the rational map by $R_{Q, \omega}$ to emphasize that the toral map is multiplication by $\omega$.

We want to be able to determine when two rational maps $R$ and $S$ are not conformally conjugate to make sure that we avoid duplication on the list of maps in Theorem 5.11. If $R$ and $S$ have different degrees, then they are not conformally conjugate. We can also use properties of the postcritical sets $P(R)$ and $P(S)$ to determine when $R$ and $S$ are not conformally conjugate. We draw a schematic diagram illustrating how the points in the postcritical set iterate. For example, the map

$$
R_{\wp, i \sqrt{2}}(z)=\frac{z^{2}+1+\sqrt{2}}{z^{2}-1-\sqrt{2}}
$$

(see Example 5.1) has four elements

$$
e_{1}=\wp\left(\frac{1}{2}\right), e_{2}=\wp\left(\frac{i \sqrt{2}}{2}\right), e_{3}=\wp\left(\frac{1+i \sqrt{2}}{2}\right) \text { and } \wp(0)=\infty,
$$

in the postcritical set. We represent the behavior of the postcritical set for the map $R_{\wp, i \sqrt{2}}(z)$ by the schematic diagram:

$$
\begin{aligned}
& e_{1} \rightarrow e_{2} \rightarrow \infty \rightarrow \infty \ldots, \\
& e_{3} \rightarrow e_{2} \rightarrow \infty \rightarrow \infty \ldots
\end{aligned}
$$

If $R$ and $S$ are rational maps with different postcritical schematic diagrams, then $R$ and $S$ are not conformally conjugate (see [Be]). If the schematic diagram representing the behavior of the postcritical set is the same for two rational maps, then more work needs to be done to determine whether or not they are conformally conjugate (see for example [Mc2] ).

We break the proof of Theorem 5.11 into cases:

5.7.1. The Weierstrass Elliptic Function $\wp$. Recall that the Weierstrass elliptic function $\wp$ identifies $u$ and $-u(\bmod \Gamma)$. There are exactly 4 points in $U_{\tau}$ where

$$
u \equiv-u(\bmod \Gamma): \frac{1}{2}, \frac{\tau}{2}, \frac{1+\tau}{2}, \text { and } 0 .
$$

Therefore, the critical values of the rational map $R_{\wp, \omega}$ are

$$
e_{1}=\wp\left(\frac{1}{2}\right), e_{2}=\wp\left(\frac{\tau}{2}\right), e_{3}=\wp\left(\frac{1+\tau}{2}\right) \text { and } \wp(0)=\infty,
$$

as these points have fewer than $d$ preimages under $R_{\wp, \omega}$.

Since we are only interested in examining measure-theoretic properties of these maps, we can restrict the period parallelogram $U_{\tau}$ further and still obtain all of the relevant measure-theoretic information about the toral endomorphism $T_{\omega}$. We use the property $\wp(u)=\wp(-u)$ to choose a subset $Y_{\wp}$ of the topological closure of $U_{\tau}$; we refer to this as Construction 1.

\section{Construction 1}

1. Pick $v$ on the boundary of $U_{\tau}$ between $u=0$ and $u=\tau$.

2. Let $w=-v+1+\tau$. Then $w$ is on the boundary of $\overline{U_{\tau}}$ between $u=1$ and $u=1+\tau$.

3. Let $l_{v w}$ be a one-dimensional curve connecting $v$ and $w$ which is symmetric with respect to the center of the period parallelogram $u=1 / 2+\tau / 2$. 


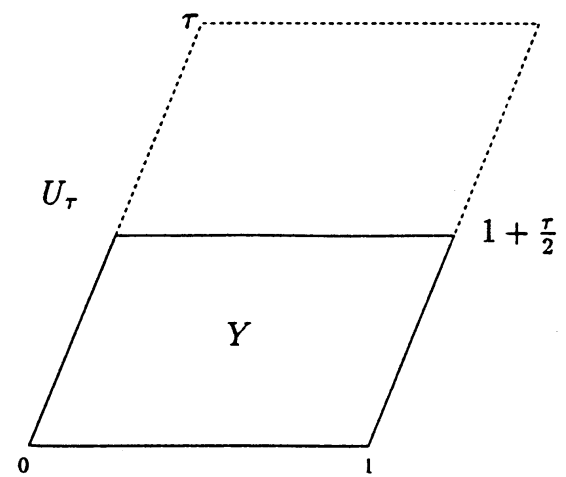

FiguRE $10 . Y$

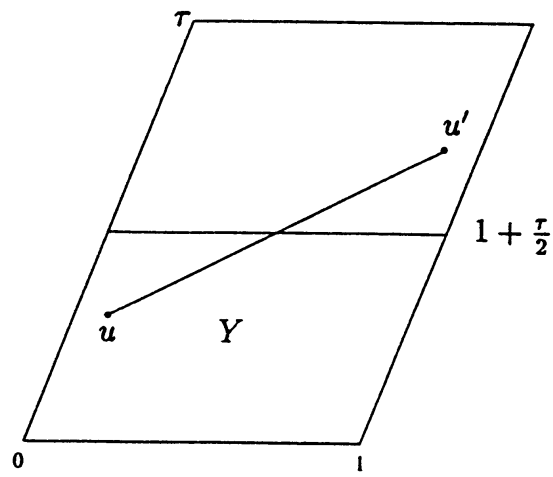

FIGURE 11. $u \in Y_{\wp}^{0}$

4. We define the boundary of $Y_{\wp}$ as follows: the boundary of $U_{\tau}$ between 0 and $v$, the curve $l_{v w}$ between $v$ and $w$, the boundary of $U_{\tau}$ between $w$ and 1 , and the boundary of $U_{\tau}$ between 1 and 0 .

Any set $Y_{\wp}$ obtained through Construction 1 satisfies the following lemma.

Proposition 5.16. If $Y_{\wp}$ is chosen through Construction 1, then there exists a set of $\nu_{\wp}$-measure 1 such that $\left.\wp\right|_{Y_{\wp}} ^{-1}(z)$ gives exactly one value in $Y_{\wp}$.

Proof. Let $Y_{\wp}^{0}$ denote the interior of $Y_{\wp}$. Clearly, $Y_{\wp}-Y_{\wp}^{0}$ has leb-measure 0, so assume that $u \in Y_{\wp}^{0}$. Then the midpoint of the line segment connecting $u$ and $u^{\prime}=-u(\bmod \Gamma)=-u+1+\tau$ is $\frac{1}{2}\left(u+u^{\prime}\right)=\frac{1}{2}(1+\tau)$, and thus $u^{\prime} \in U_{\tau}-Y_{\wp}^{0}$. Similarly, if $u \notin Y_{\wp}$ then $u^{\prime} \in Y_{\wp}^{0}$.

We want to identify $Y_{\wp}$ with a factor of $\mathbb{C} / \Gamma$ induced by the map $\wp$ up to sets of leb-measure 0, i.e. $Y_{\wp}$ is an isomorphic copy of the sphere. For the rest of this section, we denote $Y_{\wp}$ by $Y$ for simplicity of notation, but noting that $Y$ also depends on $\tau$.

Example 5.17. Define $v=\frac{\tau}{2}$ and then $w=1+\frac{\tau}{2}$. If $l_{v w}$ is a straight line connecting $v$ and $w$, then we can describe $Y$ by

$$
Y=\left\{c+d \tau \in \mathbb{C}: 0 \leq c \leq 1,0 \leq d \leq \frac{1}{2}\right\} \subset \overline{U_{\tau}}
$$

$Y$ is shown in Figure 10 .

In Figure 11] we illustrate how Proposition 5.16 applies to this $Y$.

Figure 11 shows that the midpoint of the line segment connecting $u$ and $u^{\prime}$ is $\frac{1}{2}\left(u+u^{\prime}\right)=\frac{1}{2}(1+\tau)$, and thus $u^{\prime} \in U_{\tau}-Y_{\wp}^{0}$.

Fix any $Y$ obtained through Construction 1 associated with a map $T_{\omega}$. We then obtain a map $T_{\wp, \omega}: Y \rightarrow Y$ given by

$$
T_{\wp, \omega}(u)=\left.\left.\wp\right|_{Y} ^{-1} R_{\wp, \omega}\right|_{Y}(u)=\left.\left.\wp\right|_{Y} ^{-1} \wp T_{\omega} \wp^{-1} \wp\right|_{Y}(u)
$$


and the following diagram commutes:

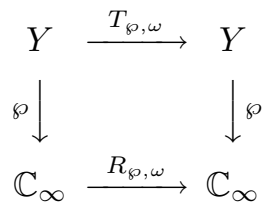

We note that for all $n>0$, we have

$$
T_{\wp, \omega}^{n}(u)=\left.\left.\wp\right|_{Y} ^{-1} \wp T_{\omega}^{n} \wp^{-1} \wp\right|_{Y}(u) .
$$

We use the appropriate $\sigma$-algebra and measure on $Y \subset \overline{U_{\tau}}$ which makes it a factor of $\mathbb{C} / \Gamma$. Let $\mathcal{C}$ be the Borel $\sigma$-algebra on $Y$, and let $\mu_{\wp}$ be normalized Lebesgue measure on $Y$, which is given by $\mu_{\wp}(E)=2 \operatorname{leb}(E)$ for all $E \in \mathcal{C}$. Then $T_{\wp, \omega}$ is measure-preserving with respect to $\mu_{\wp}$. We thus have the following lemma.

Lemma 5.18. $\left(Y, \mathcal{C}, \mu_{\wp}, T_{\wp, \omega}\right) \cong\left(\mathbb{C}_{\infty}, \mathcal{C}_{2}, \nu_{\wp}, R_{\wp, \omega}\right)$.

To prove our theorem, it suffices to show that $\left(Y, \mathcal{C}, \mu_{\wp}, T_{\wp, \omega}\right)$ is one-sided Bernoulli of entropy $\log \left(\operatorname{deg} R_{\wp, \omega}\right)$. We note that the isomorphism in Lemma 5.18 extends to other tori $\mathbb{C} / \Gamma^{\prime}$ where $T_{\omega}$ is defined on the torus $\mathbb{C} / \Gamma^{\prime}$ (see $[\mathrm{B}]$ or $[\mathrm{B}-\mathrm{K}]$ ).

Proof of Theorem 5.11(1). For any $d>1$, write $d=c^{2} q$ where $c, q$ are positive integers, and $q$ has no square factors other than 1 . We define the lattice $\Gamma=\Gamma_{c i \sqrt{q}}=$ $[1, c i \sqrt{q}]$, and then $T_{c i \sqrt{q}}(u)=i c \sqrt{q} u$ is an endomorphism of $\mathbb{C} / \Gamma$. Therefore, $R_{\wp, c i \sqrt{q}}(z)=\wp \circ T_{c i \sqrt{q}} \circ \wp^{-1}(z)$ is a degree $d$ rational map of the sphere.

We describe the behavior of the postcritical set of this family of maps. Let $e_{1}=\wp\left(\frac{1}{2}\right), e_{2}=\wp\left(\frac{c i \sqrt{q}}{2}\right), e_{3}=\wp\left(\frac{1+c i \sqrt{q}}{2}\right)$ and $\wp(0)=\infty$. If $d$ is even,

$$
\begin{aligned}
& e_{1} \rightarrow e_{2} \rightarrow \infty \rightarrow \infty \ldots, \\
& e_{3} \rightarrow e_{2} \rightarrow \infty \rightarrow \infty \ldots
\end{aligned}
$$

If $d$ is odd,

$$
\begin{gathered}
e_{1} \rightarrow e_{2} \rightarrow e_{1} \ldots, \\
e_{3} \rightarrow e_{3} \ldots, \\
\infty \rightarrow \infty \ldots .
\end{gathered}
$$

Let $Y=\left\{c+d \tau \in \mathbb{C}: 0 \leq c \leq 1,0 \leq d \leq \frac{1}{2}\right\}$, let $\mathcal{C}$ be the Borel $\sigma$-algebra on $Y$, let $\mu_{\wp}$ be normalized Lebesgue measure in $Y$, and let

$$
T_{\wp, i \sqrt{2}}(u)=\left.\wp\right|_{Y} ^{-1} R_{\wp,\left.i \sqrt{2} \wp\right|_{Y}(u) .}
$$

Then $\left(Y, \mathcal{C}, \mu_{\wp}, T_{\wp, i \sqrt{2}}\right)$ is measure-theoretically isomorphic to the rational map of the sphere $R_{\wp, i \sqrt{2}}(z)$ with its measure of maximal entropy. Define the partition $\mathcal{P}_{i \sqrt{2}}=\left\{A_{0}, A_{1}\right\}$ of $Y$ as follows:

$$
\begin{aligned}
& A_{0}=\left\{u=(a, b) \in Y: a \leq \frac{1}{2}\right\}, \\
& A_{1}=\left\{u=(a, b) \in Y: a>\frac{1}{2}\right\} .
\end{aligned}
$$




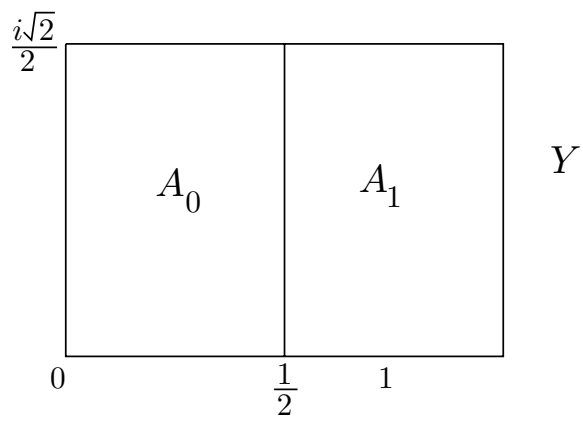

FIgURE $12 . \mathcal{P}_{i \sqrt{2}}$

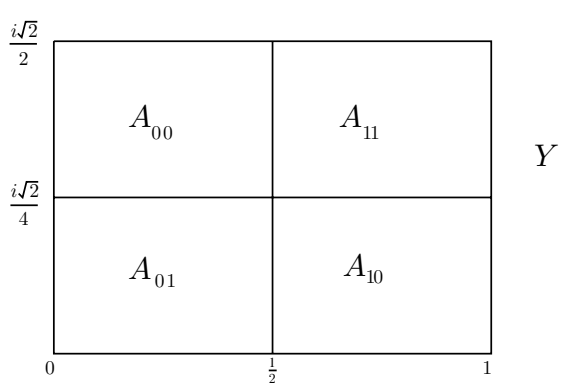

Figure 13. $\mathcal{P}_{i \sqrt{2}}^{1}$

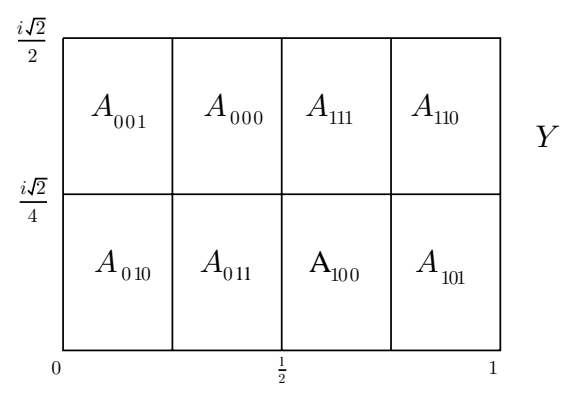

FIGURE 14. $\mathcal{P}_{i \sqrt{2}}^{2}$

Figure 12 shows an illustration of $\mathcal{P}_{i \sqrt{2}}$. We use the partition to code the dynamical system by defining $\varphi: Y \rightarrow X_{2}^{+}$by

$$
\varphi(u)=\left(c_{0}, c_{1}, c_{2}, \ldots\right) \text { where } T_{\wp, i \sqrt{2}}^{j}(u) \in A_{c_{j}} .
$$

Let $\mathcal{P}_{i \sqrt{2}}^{k}=\bigvee_{j=0}^{k} T_{\wp, i \sqrt{2}}^{-j}\left(\mathcal{P}_{i \sqrt{2}}\right)$. We illustrate $\mathcal{P}_{i \sqrt{2}}^{1}=\mathcal{P}_{i \sqrt{2}} \bigvee T_{\wp, i \sqrt{2}}^{-1} \mathcal{P}_{i \sqrt{2}}$ in Figure 13, and $\mathcal{P}_{i \sqrt{2}}^{2}$ in Figure 14

It is straightforward to prove that all $P \in \mathcal{P}_{i \sqrt{2}}^{k}$ are congruent rectangles and thus have the same diameter. Further, we can calculate for any $P \in \mathcal{P}_{i \sqrt{2}}^{k}$, the $\operatorname{diam}(P)=\frac{\sqrt{3}}{\sqrt{2}^{2+2}}$. Therefore,

$$
\lim _{k \rightarrow \infty}\left(\sup _{P \in \mathcal{P}_{i \sqrt{2}}^{k}} \operatorname{diam}(P)\right)=\lim _{k \rightarrow \infty} \frac{\sqrt{3}}{\sqrt{2}^{k+2}}=0 .
$$

Therefore, $\varphi$ is an isomorphism.

For $d>2$ we split the proof into the cases of $d$ even and $d$ odd. Recall that $\omega=i c \sqrt{q}$ throughout.

Extension to Maps of Even Degree. If $d=c^{2} q$ is even, then using $\Gamma=[1, c i \sqrt{q}]$, we have that $T_{c i \sqrt{q}}(u)=c i \sqrt{q} u$ is an endomorphism of $\mathbb{C} / \Gamma$ of degree $d=c^{2} q$. We use the following partition $\mathcal{P}_{c i \sqrt{q}}=\left\{A_{0}, A_{1}, \ldots, A_{d-1}\right\}$ of $Y$ (see Figure [15) where 


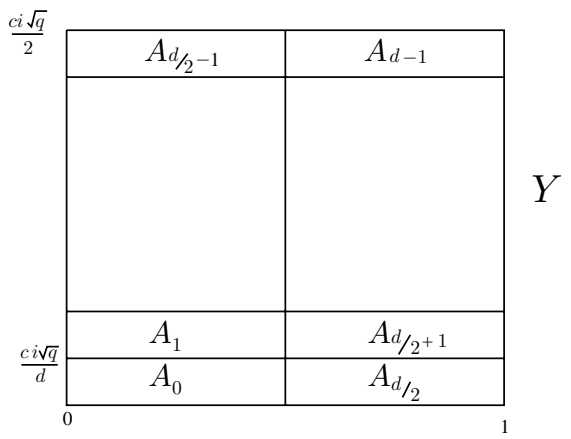

FIGURE 15. $\mathcal{P}_{c i \sqrt{q}}$ with $d$ even

$$
\left.\mu_{\wp}\left(A_{j}\right)=1 / d: \quad \begin{array}{rl}
u=(a, b) \in Y: & a \leq \frac{1}{2}, \frac{c j \sqrt{q}}{d} \leq b<\frac{c(j+1) \sqrt{q}}{d} \\
& \text { when } j=0,1, \ldots \frac{d}{2}-1 \\
u=(a, b) \in Y: & a>\frac{1}{2}, \frac{c\left(j-\frac{d}{2}\right) \sqrt{q}}{d} \leq b<\frac{c\left(j-\frac{d}{2}+1\right) \sqrt{q}}{d} \\
\text { when } j=\frac{d}{2}, \ldots, d-1
\end{array}\right\} .
$$

Again, all $P \in \mathcal{P}_{c i \sqrt{q}}^{k}$ are congruent rectangles and thus have the same diameter. Further, $\bigvee_{j=0}^{k} T_{\wp, c i \sqrt{q}}^{-j}\left(\mathcal{P}_{c i \sqrt{q}}\right)$ cuts every $P \in \mathcal{P}_{c i \sqrt{q}}^{k-1}$ into $d$ congruent rectangles each of which is similar to $P$. An argument identical to the degree two case gives that the rational map $R_{\wp, c i \sqrt{q}}(z)=\wp \circ T_{c i \sqrt{2}} \circ \wp^{-1}(z)$ is isomorphic to a one-sided Bernoulli shift of entropy $\log d$.

Extension to Maps of Odd Degree. We use Construction 1 to choose a different representative $Y$ of the sphere in the case when $d=c^{2} q$ is odd. If $v=\frac{c i(d+1) \sqrt{q}}{2 d}$, then Construction 1 gives the region $Y$ (illustrated in Figure 16) defined by

$$
\begin{aligned}
Y= & \left\{u=(a, b) \in U_{c i \sqrt{q}}: a \leq \frac{1}{2} \text { and } b<\frac{c(d+1) \sqrt{q}}{2 d}\right\} \\
& \cup\left\{u=(a, b) \in U_{c i \sqrt{q}}: a \geq \frac{1}{2} \text { and } b<\frac{c(d-1) \sqrt{q}}{2 d}\right\} .
\end{aligned}
$$

As before, Proposition 5.16 implies that we can observe the important measuretheoretic aspects of the rational map $R_{\wp, c i \sqrt{q}}$ by examining the map $T_{\wp, c i \sqrt{q}}$ on $Y$. Let $\mathcal{P}_{c i \sqrt{q}}=\left\{A_{0}, A_{1}, \ldots, A_{d-1}\right\}$ be the following partition of $Y$ into $d$ congruent pieces:

$$
A_{j}=\left\{\begin{array}{l}
u=(a, b) \in Y: a \leq \frac{1}{2}, \frac{c(j) \sqrt{q}}{d} \leq b<\frac{c((j+1)) \sqrt{q}}{d} \\
\text { when } j=0,1, \ldots, \frac{d-1}{2}-1 . \\
u=(a, b) \in Y: a>\frac{1}{2}, \frac{c\left(j-\frac{d-1}{2}\right) \sqrt{q}}{d} \leq b<\frac{c\left(j-\frac{d-3}{2}\right) \sqrt{q}}{d} \\
\text { when } j=\frac{d-1}{2}, \ldots, d-2 . \\
u=(a, b) \in Y: b>\frac{c j \sqrt{q}}{2 d} \\
\text { when } j=d-1 .
\end{array}\right\} .
$$

We include an illustration of the general partition $\mathcal{P}_{c i \sqrt{q}}$ when $d$ is odd in Figure 17 


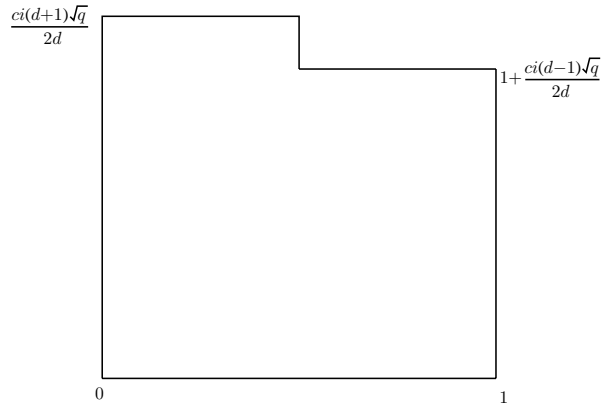

Figure 16. $Y \subset U_{c i \sqrt{q}}$ with $d$ odd

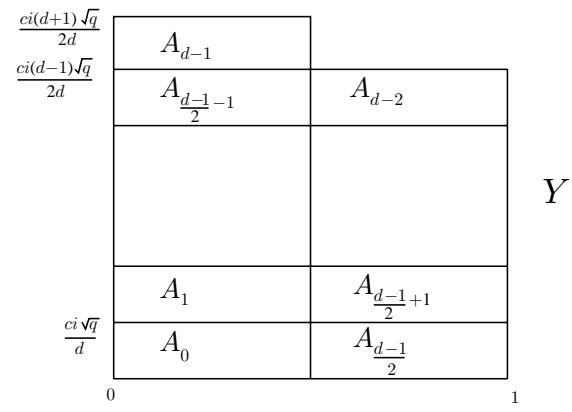

Figure 17. $\mathcal{P}_{c i \sqrt{q}}$ with $d$ odd

When $d$ is odd, all $P$ in $\mathcal{P}_{c i \sqrt{q}}^{k}$ have area $\frac{c \sqrt{q}}{2 d^{k+1}}$ and we have

$$
\sup _{P \in \mathcal{P}_{c i}^{n} \sqrt{q}}(\operatorname{diam}(P)) \rightarrow 0 .
$$

Therefore, the rational map $R_{\wp, c i \sqrt{q}}(z)=\wp \circ T_{c i \sqrt{q}} \circ \wp^{-1}(z)$ is isomorphic to a one-sided Bernoulli shift of entropy $\log d$. We have thus completed the proof of Theorem 5.11(1).

We use the same procedure to prove that every rational map listed in Theorem 5.11 is one-sided Bernoulli. Given a toral endomorphism $T_{\omega}$ on $\mathbb{C} / \Gamma$ and an elliptic function $Q \in\left\{\wp, \wp^{2}, \wp^{\prime},\left(\wp^{\prime}\right)^{2}\right\}$, we find a set $Y_{Q}$ and a map $T_{Q, \omega}$ which is a factor of $T_{\omega}$ that is measure-theoretically isomorphic to $R_{Q, \omega}$. We then find a partition $\mathcal{P}_{\omega}$ of $Y_{Q}$ into $d$ congruent pieces which generates independently with respect to $T_{Q, \omega}$. Therefore, using Theorem 2.1, we have an isomorphism to a one-sided Bernoulli shift of entropy $\log d$. The details of the arguments vary in one of two ways. First, we may need to choose a different representative $Y_{Q}$ depending on the particular toral endomorphism or elliptic function with which we are working. Second, we need to find an independent partition which generates with respect to $T_{Q, \omega}$. While there are many different choices for $Y_{Q}$, finding an independent generating partition can be quite challenging. In some cases, the choice of $Y_{Q}$ and $\mathcal{P}_{\omega}$ must be fractal.

To prove the rest of the cases listed in Theorem 5.11 we describe our choice of $Y$, define our partition $\mathcal{P}_{\omega}$, and calculate the diameter of an element in the $k$ th refinement of the partition.

Proof of Theorem $\left[5.11(2)\right.$. Let $\Gamma=[1, \rho]$ where $\rho=e^{\frac{i \pi}{3}}$. Then $T_{n+n i \sqrt{3}}(u)=$ $n(1+i \sqrt{3}) u$ is an endomorphism of $\mathbb{C} / \Gamma$ of degree $4 n^{2}$.

We define $Y=\left\{u=(a, b) \in U_{\rho}: b \leq-\sqrt{3}(a-1)\right\}$ through Construction 1. We begin by proving the theorem in the case where $n=1$ (a degree four map). Let $\mathcal{P}_{1+i \sqrt{3}}=\left\{A_{0}, A_{1}, A_{2}, A_{3}\right\}$ be the partition of $Y$ illustrated in Figure 18 ,

We can calculate for any $P \in \mathcal{P}_{1+i \sqrt{3}}^{k}$ the diam $P=\frac{1}{2^{k+1}}$. Therefore,

$$
\lim _{k \rightarrow \infty}\left(\sup _{P \in \mathcal{P}_{1+i \sqrt{3}}^{k}} \operatorname{diam}(P)\right)=\lim _{k \rightarrow \infty} \frac{1}{2^{k+1}}=0 .
$$

Applying Theorem [2.1, $\varphi$ is an isomorphism, and we have completed the proof in the case $n=1$. The general degree $4 n$ result follows from a similar partition. 


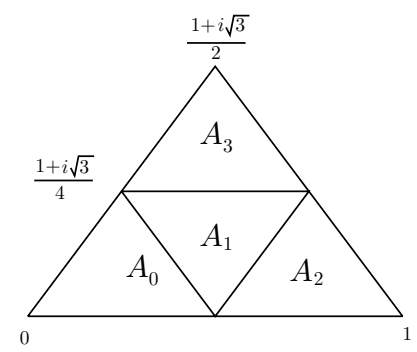

FiguRE 18. $\mathcal{P}_{1+i \sqrt{3}}$

We note that the postcritical set of the family $R_{\wp, n+n i \sqrt{3}}$ behaves in the following manner, where $e_{1}, e_{2}$, and $e_{3}$ are given in eq. (16).

$$
\begin{aligned}
& e_{1} \rightarrow \infty \rightarrow \infty \ldots, \\
& e_{2} \rightarrow \infty \rightarrow \infty \ldots, \\
& e_{3} \rightarrow \infty \rightarrow \infty \ldots
\end{aligned}
$$

Proof of Theorem 5.11(3). Up to this point in our investigation, the toral endomorphisms that we have examined have been rotations through rational angles and expansions by a constant greater than one. This rotation through a rational angle facilitated our construction of a finite independent generating partition on $Y$. However, many toral endomorphisms involve a rotation through an irrational angle with an expansion. In this section, we examine toral endomorphisms given by the equation $T_{n+l i}(u)=(n+l i) u$, where exactly one of $l, n$ are odd, on the lattice $\Gamma=[1, i]$. If $n$ is odd and $l$ is even, the resulting rational map of the sphere $R_{\wp, n+l i}(z)=\wp \circ T_{n+l i} \circ \wp^{-1}(z)$ has postcritical set consisting of four fixed critical values:

$$
\begin{aligned}
& e_{1} \rightarrow e_{1} \ldots, \\
& e_{2} \rightarrow e_{2} \ldots, \\
& e_{3} \rightarrow e_{3} \ldots, \\
& \infty \rightarrow \infty \ldots
\end{aligned}
$$

Thus these maps are not conformally conjugate to any of the rational maps previously discussed in this chapter.

If $n$ is even and $l$ is odd, the postcritical set behaves as follows:

$$
\begin{gathered}
e_{1} \rightarrow e_{2} \rightarrow e_{1} \ldots, \\
e_{3} \rightarrow e_{3} \ldots, \\
\infty \rightarrow \infty \ldots
\end{gathered}
$$

We begin by discussing the simplest map in this family, the degree five toral endomorphism $T_{1+2 i}(u)=(1+2 i) u$. Figure 19 represents an illustration of $U_{1+2 i}$, the planar representation of the torus that we will use. Figure 19] is drawn using an iterative procedure defined in [I] and [I-O], and the reader should refer to those 
papers for the details. The fractal boundary enables us to find an independent generating partition for the map $T_{1+2 i}$ on the torus. We use $\mathcal{C}_{1}$ to denote the Borel $\sigma$-algebra in $U_{1+2 i}$ and $l e b$ to denote normalized Lebesgue measure in $U_{1+2 i}$.

We illustrate the independent generating partition $\mathcal{Q}=\left\{B_{0}, B_{1}, B_{2}, B_{3}, B_{4}\right\}$ constructed in [I] and [I-O] in Figure 20. Note that each $B_{j}$ is a region that is similar to $U_{1+2 i}$. We cannot use this partition exactly to create a partition on the sphere because $\mathcal{Q}$ does not respect the relationship $u \equiv-u(\bmod \Gamma)$ given by the $\wp$-function. However, we can use the boundaries of the partition $\mathcal{Q}$ and its refinements $\mathcal{Q}^{k}=\bigvee_{j=0}^{k} T_{1+2 i}^{-j}(\mathcal{Q})$ to define a region $Y$ which is isomorphic to the sphere. We make this definition iteratively.

Let $p$ be the point where the boundaries of $B_{0}, B_{1}$, and $B_{2}$ intersect. We begin with the section of the boundary of region $B_{1}$ that lies between the points $p$ and $q$ in Figure 21 Call this section $L_{1}$. In addition, we select all points in $U_{1+2 i}$ that are reflected through the point $1 / 2+i / 2$, denoted $L_{2}$. (Notice that these are just the points $-u(\bmod \Gamma)$ where $u$ is a point on $L_{1}$.) Next, we examine the refinement $\mathcal{Q}^{1}$. We select the region on the boundary of the partition $\mathcal{Q}^{1}$ that lies between the points $q$ and $r$ (denoted $L_{3}$ ), and the reflection of these points through $1 / 2+i / 2$ (denoted $L_{4}$ ). See Figure 22 for an illustration of $q$ and $r$. We continue this process indefinitely. That is, after we find $L_{2 k+1}$ we choose a section of the boundary of the partition $\mathcal{Q}_{k+1}$ that intersects $L_{2 k+1}$ and we denote this region by $L_{2 k+3}$. We also choose the reflection of $L_{2 k+3}$ through the center of the period parallelogram and call it $L_{2 k+4}$. In this way we obtain $L=\bigcup L_{j}$, which will split $U_{1+2 i}$ into two symmetric pieces. Let $Y$ be the region lying below $L$. We have defined $Y$ to satisfy Proposition 5.16, it is clear that $u \in Y \backslash \partial Y$ if and only if $-u(\bmod \Gamma) \in U_{i}^{\prime} \backslash Y$. Thus on a set of $\nu_{\wp}$-measure one, every $z \in \mathbb{C}_{\infty}$ has a unique representative in $Y$. We illustrate the resulting region $Y$ in Figure 23.

We split each partition piece $B_{j}$ into two pieces in the same way that $U_{1+2 i}$ is split by $L$. Namely, we copy segments $L^{\prime}=L / \sqrt{5}$ into each $B_{j}$ so that they are split into two pieces. We take the resulting five pieces that lie in $Y$ as our partition $\mathcal{P}_{1+2 i}=\left\{A_{0}, A_{1}, A_{2}, A_{3}, A_{4}\right\}$ (see Figure 24). We know that $\mathcal{Q}$ is an independent generating partition. We have split the atoms of $\mathcal{Q}$ into two congruent pieces using refinements of the independent partitions $\mathcal{Q}^{k}$. Thus each atom of $\mathcal{P}_{1+2 i}^{k}$ is exactly half of an atom of $\mathcal{Q}_{k}$ and is congruent to $Y$. Since the measure $\mu_{\wp}$ is normalized Lebesgue measure in $Y$, the partitions $\mathcal{P}_{1+2 i}^{k}$ are independent. Further, each application of the map $T_{1+2 i}^{-1}$ is a rotation by $-\tan ^{-1}(2)$ and a contraction by $\frac{1}{\sqrt{5}}$. We can calculate for any $P \in \mathcal{P}_{1+2 i}^{k}$,

$$
\operatorname{diam} P \leq \frac{1}{\sqrt{5}^{k}}\left(\sqrt{\frac{2(6+2 \sqrt{5})}{5}}\right)
$$

Applying Theorem 2.1 $\varphi$ is an isomorphism and we have completed the proof in the case $n=1, l=2$.

The proof when $n, l>1$ and exactly one of $l, n$ is odd can be completed in the same way. We use the generating partition $\mathcal{Q}=\left\{B_{0}, B_{1}, \ldots, B_{d-1}\right\}$ (where $d=l^{2}+n^{2}$ ) from Theorem [5.10 for the endomorphism $T_{n+l i}$ first to define the region $Y$ which is isomorphic to the sphere. Then we modify the partition $\mathcal{Q}$ by splitting each $B_{j}$ into two pieces in the same manner that $U_{i}^{\prime}$ was split by $L$. This 


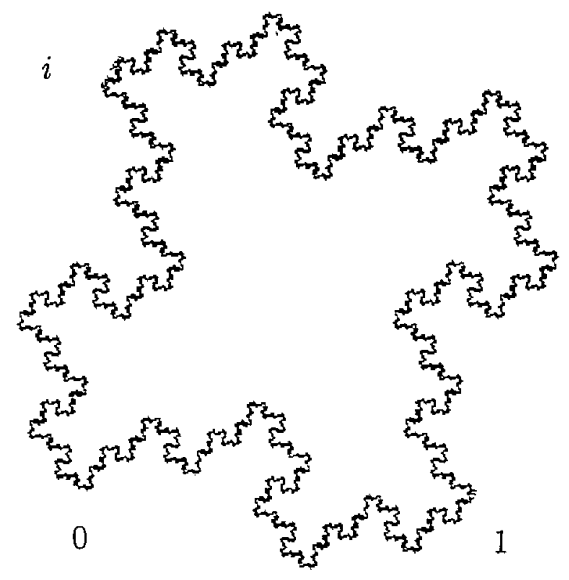

FigURE 19. $U_{1+2 i}$

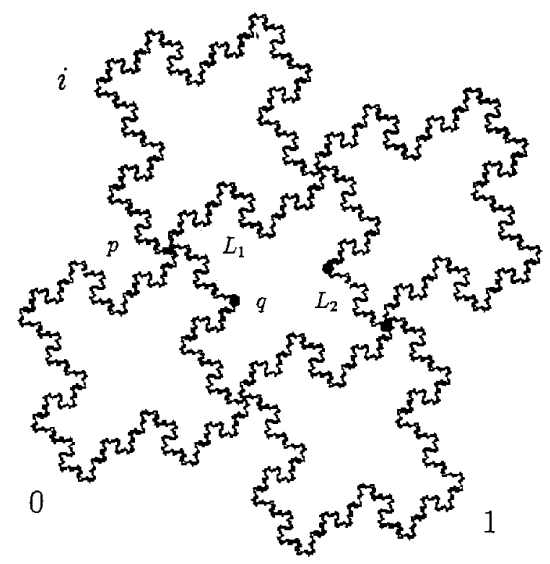

FiguRE 21. $L_{1}$ and $L_{2}$

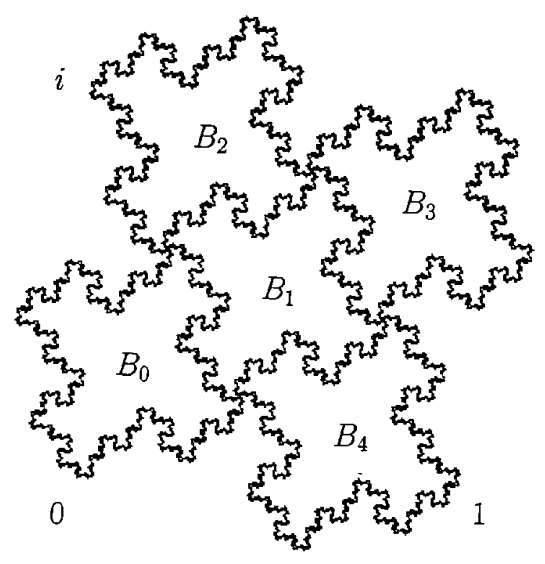

Figure 20. $\mathcal{Q}$

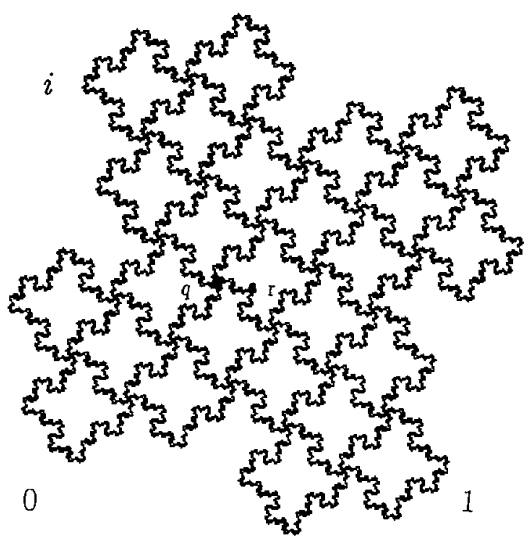

FIGURE 22. $q$ and $r$

gives an independent generating partition on $Y$, and hence $R_{\wp, n+l i}$ is one-sided Bernoulli with respect to $\nu_{\wp}$.

5.7.2. The Elliptic Function $\wp^{2}$. Here we examine rational maps which are factors of toral endomorphisms via the elliptic function $\wp^{2}$. We begin with the lattice $\Gamma_{i}=[1, i]$. Any map of the form $T_{a+i b}(u)=(a+i b) u$, where $a, b \in \mathbb{Z}$, is an endomorphism of $\mathbb{C} / \Gamma$. We define a rational map of the sphere by the equation $R_{\wp^{2}, a+i b}(z)=\wp^{2} \circ T_{a+i b} \circ\left(\wp^{2}\right)^{-1}(z)$. Let $\nu_{\wp^{2}}(F)=\operatorname{leb}\left(\left(\wp^{2}\right)^{-1}(F)\right)$ for all $F \in \mathcal{C}_{2}$.

The images of the three points $\frac{1}{2}, \frac{1+i}{2}$, and 0 under the elliptic function $\wp^{2}$ form the set of critical values of $R_{\wp^{2}, a+i b}$, as these points have fewer than $d$ preimages under $R_{\wp^{2}, a+i b}$. We denote the critical values of the rational map $R_{\wp^{2}, a+i b}$ as follows:

$$
e_{1}=\wp^{2}\left(\frac{1}{2}\right), e_{2}=\wp^{2}\left(\frac{1+i}{2}\right), \text { and } \wp^{2}(0)=\infty .
$$




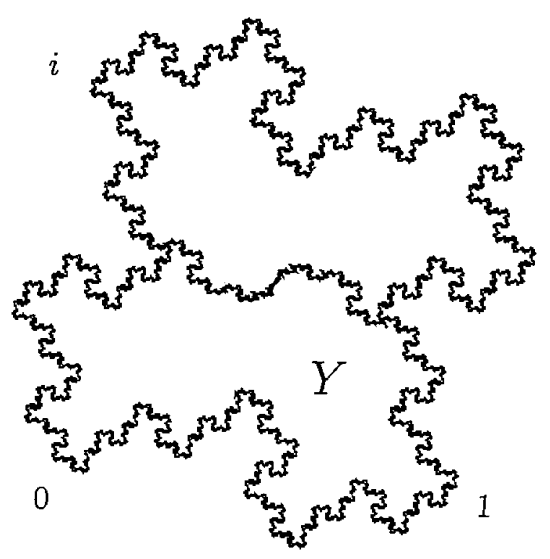

FigURE 23. $Y$

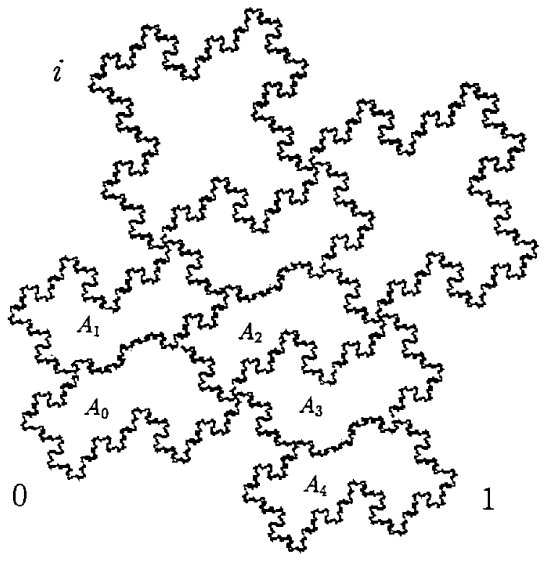

FIGURE 24. $\mathcal{P}_{1+2 i}$

We note that the postcritical set of $R_{\wp^{2}, a+i b}$ is $\left\{e_{1}, e_{2}, \infty\right\}$, hence none of the resulting rational maps $R_{\wp^{2}, a+i b}$ are conjugate via a Möbius transformation to any in Section 5.7.1 or [B], [B-K].

Again, we restrict the region $U_{1+i}$ to a smaller region which still retains all of the relevant measure-theoretic information about the map $T_{a+i b}$. We define the region $Y_{\wp}$ from the region $Y_{\wp}$ in Construction 2.

\section{Construction 2}

1. Pick $v, w$, and $l_{v w}$ as in Construction 1 (see eq. (17)).

2. Define $l_{v w}^{\prime}$ by rotating $l_{v w}$ by $\pi / 2$ around the midpoint of the period parallelogram $u=1 / 2+i / 2$. Let $w^{\prime}$ be the intersection of $l_{v w}^{\prime}$ and the positive $x$-axis.

3. The region $Y_{\wp^{2}}$ has boundary as follows: The boundary of $U_{i}$ from 0 to $v$, the curve $l_{v w}$ from $v$ to $1 / 2+i / 2$, the curve $l_{v w}^{\prime}$ from $1 / 2+i / 2$ to $w^{\prime}$, and the boundary of $U_{i}$ from $w^{\prime}$ to 0 .

Let $u$ be in the interior of $Y_{\wp^{2}}$. Then $i u,-u,-i u \in U_{\tau} \backslash Y_{\wp^{2}}$. Thus on a set of $\nu_{\wp^{2}}$-measure one, $\left.\wp^{2}\right|_{Y_{\wp^{2}}} ^{-1}(z)$ gives exactly one value in $Y_{\wp^{2}}$. Therefore, we obtain a well-defined map $T_{\wp^{2}, a+i b}: Y_{\wp^{2}} \rightarrow Y_{\wp^{2}}$ by

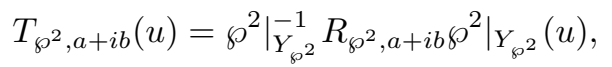

and the following diagram commutes:

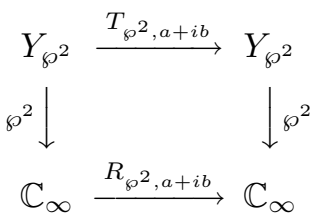

Let $\mathcal{C}$ be the Borel $\sigma$-algebra on $Y_{\wp^{2}}$ and $\mu_{\wp^{2}}$ be normalized Lebesgue measure on $Y_{\wp^{2}}$, which is given by $\mu_{\wp^{2}}(E)=4 l e b(E)$ for all $E \in \mathcal{C}$. Then $T_{\wp^{2}, a+i b}$ is measurepreserving with respect to $\mu_{\wp^{2}}$. Again, we have the following reduction lemma. For simplicity of notation, we denote $Y_{\wp^{2}}$ by $Y$.

Lemma 5.19. $\left(Y, \mathcal{C}, \mu_{\wp^{2}}, T_{\wp^{2}, a+i b}\right) \cong\left(\mathbb{C}_{\infty}, \mathcal{C}_{2}, \nu_{\wp^{2}}, R_{\wp^{2}, a+i b}\right)$. 


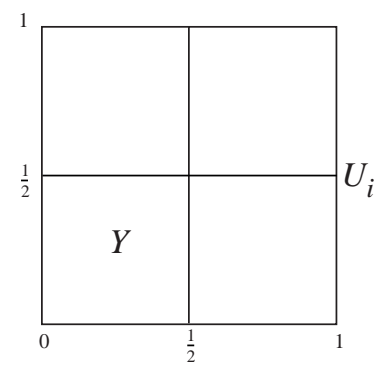

FiguRE 25. $Y$

Example 5.20. Define $v, w$, and $l_{v w}$ as in Example 5.17 on the period parallelogram $U_{i}$. Then using Construction 2 we obtain the region $Y$ defined by $Y=\left\{u=(x, y) \in \mathbb{C}: y \leq \frac{1}{2}\right.$ and $\left.x \leq \frac{1}{2}\right\} \subset U_{i}$ and illustrated in Figure 25.

Proof of Theorem $5.11(4),(5)$. We begin our investigation of rational maps with $(2,4,4)$ orbifolds with a study of toral endomorphisms of the form $T_{n}(u)=n u$ or $T_{n i}(u)=n i u$ where $|n|=2,3,4, \ldots$ We obtain that $R_{\wp^{2}, n}(z)=\wp^{2}{ }_{\Gamma} \circ T_{n} \circ \wp^{2}{ }_{\Gamma}^{-1}(z)$ is a degree $n^{2}$ rational map of the sphere.

We describe the behavior of the postcritical set of these maps. Let $e_{1}=\wp^{2}\left(\frac{1}{2}\right)$, $e_{2}=\wp^{2}\left(\frac{1+i}{2}\right)$ and $\wp^{2}(0)=\infty$. Then if $n$ is even, we have

$$
\begin{aligned}
& e_{1} \rightarrow \infty \rightarrow \infty \ldots, \\
& e_{2} \rightarrow \infty \rightarrow \infty \ldots
\end{aligned}
$$

If $n$ is odd, the postcritical set consists of three fixed critical values:

$$
\begin{aligned}
& e_{1} \rightarrow e_{1} \ldots, \\
& e_{2} \rightarrow e_{2} \ldots, \\
& \infty \rightarrow \infty \ldots
\end{aligned}
$$

Note that $R_{\wp^{2}, n}$ and $R_{\wp^{2}, n i}$ are conformally conjugate maps of the sphere since $n z$ is identified with niz under $\wp^{2}$.

We partition the set $Y$ given in Example 5.20 into $n^{2}$ congruent pieces by $\mathcal{P}_{n}=$ $\left\{A_{1}, \ldots, A_{n^{2}}\right\}$ as follows:

$$
\begin{aligned}
A_{k+j n} & =\left\{u=(a, b) \in Y: \frac{k}{2 n} \leq a \leq \frac{k+1}{2 n}\right. \text { and } \\
\frac{j}{2 n} & \left.\leq b \leq \frac{j+1}{2 n} \text { when } k, j=0,1, \ldots, n-1\right\} .
\end{aligned}
$$

We include an illustration of the general partition $\mathcal{P}_{n}$ of $Y$ in Figure 26

Let $\mathcal{P}_{n}^{k}=\bigvee_{j=0}^{k} T_{\wp^{2}, n}^{-j}\left(\mathcal{P}_{n}\right)$. Then $\bigvee_{j=0}^{k} T_{\wp^{2}, n}^{-j}\left(\mathcal{P}_{n}\right)$ cuts every $P \in \mathcal{P}_{n}^{k-1}$ into $n^{2}$ squares of side length $\frac{1}{2 n^{k+1}}$ and diameter $\frac{\sqrt{2}}{2 n^{k+1}}$. Applying Theorem 2.1, $\varphi$ is an isomorphism. 


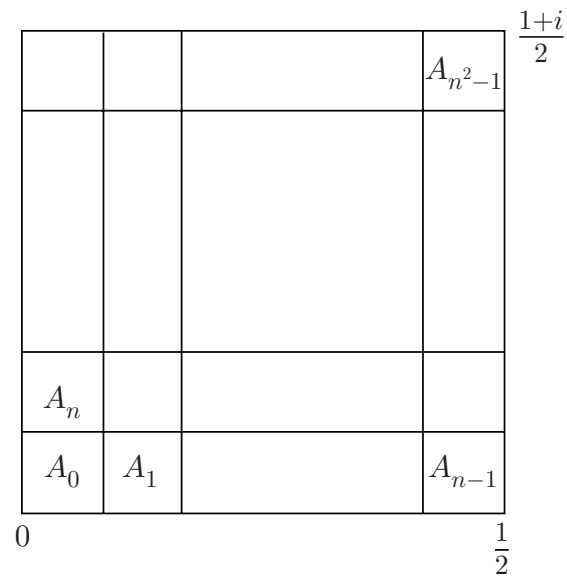

FIGURE $26 . \mathcal{P}_{n}$

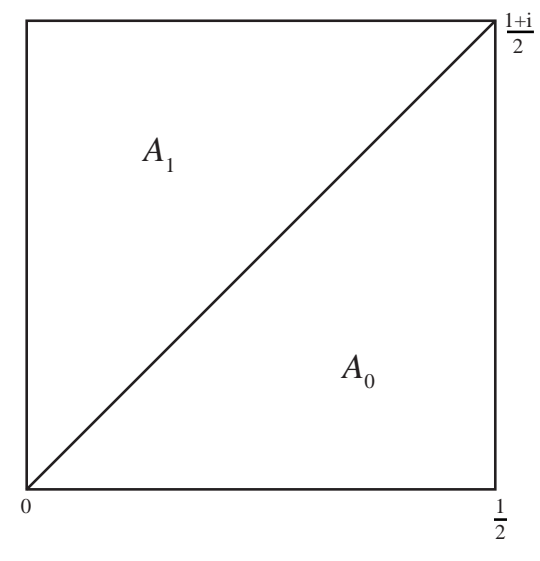

Figure 27. $\mathcal{P}_{1+i}$

Proof of Theorem $5.11(6)$. We continue our investigation of rational maps that are factors of toral endomorphisms via the elliptic function $\wp^{2}$. Let $T_{n+n i}(u)=(n+n i) u$ be a toral endomorphism of $\mathbb{C} / \Gamma$. Then the rational map of the sphere defined by $R_{\wp^{2}, n+n i}(z)=\wp^{2} \circ T_{n+n i} \circ\left(\wp^{2}\right)^{-1}(z)$ has degree $2 n^{2}$. The postcritical schematic diagram of the rational map $R_{\wp^{2}, n+n i}$ is as follows:

If $n$ is odd, then

$$
e_{1} \rightarrow e_{2} \rightarrow \infty \rightarrow \infty \ldots
$$

If $n$ is even, then

$$
\begin{aligned}
& e_{1} \rightarrow \infty \rightarrow \infty \ldots, \\
& e_{2} \rightarrow \infty \rightarrow \infty \ldots .
\end{aligned}
$$

By examining the degrees, the resulting rational map $R_{\wp^{2}, n+n i}$ is not conjugate via a Möbius transformation to any of the maps previously discussed.

Figure 27 illustrates the following partition $\mathcal{P}_{1+i}=\left\{A_{0}, A_{1}\right\}$ of $Y$, where

$$
\begin{aligned}
& A_{0}=\left\{u=(a, b) \in Y_{\wp^{2}}: b \leq a\right\}, \\
& A_{1}=\left\{u=(a, b) \in Y_{\wp^{2}}: b>a\right\} .
\end{aligned}
$$

It is straightforward to calculate for any $P \in \mathcal{P}_{1+i}^{k}$ the diameter of $P$ is $\frac{1}{\sqrt{2}^{k+1}}$. Theorem 2.1 implies that $\varphi$ is an isomorphism. The partitions $\mathcal{P}_{n+n i}$ are constructed similarly, with every $P \in \mathcal{P}_{n+n i}^{k}$ having diameter $\frac{1}{(n \sqrt{2})^{k+1}}$.

Proof of Theorem 5.11(7). We examine the toral endomorphism $T_{1+2 i}(u)=$ $(1+2 i) u$. The resulting rational map of the sphere is given by $R_{\wp^{2}, 1+2 i}(z)=\wp^{2} \circ$ $T_{1+2 i} \circ\left(\wp^{2}\right)^{-1}(z)$, and the postcritical set iterates as follows:

$$
\begin{aligned}
& e_{1} \rightarrow e_{1} \ldots, \\
& e_{2} \rightarrow e_{2} \ldots, \\
& \infty \rightarrow \infty \ldots
\end{aligned}
$$




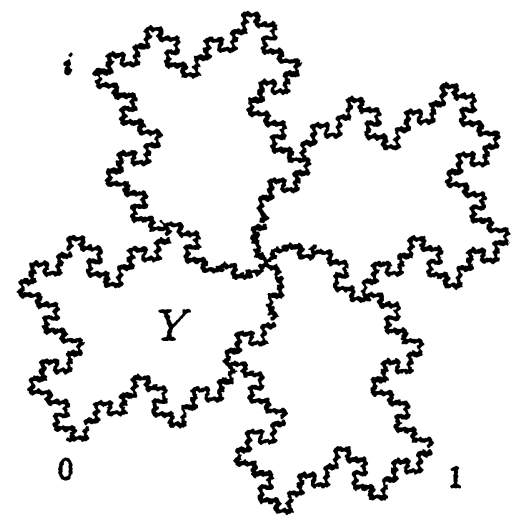

FiguRE 28. $Y$

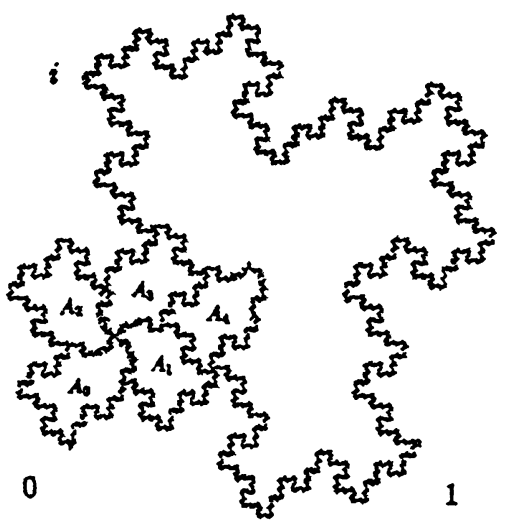

FiguRE 29. $\mathcal{P}_{1+2 i}$

Since the degree of this map (which is 5) is different than those studied in Section 5.7.2, this map is not conformally conjugate to any rational map discussed previously. We note that $\left(\mathbb{C}_{\infty}, \mathcal{C}_{2}, \nu_{\wp^{2}}, R_{\wp^{2}, 1+2 i}\right)$ is a two-point factor of $\left(\mathbb{C}_{\infty}, \mathcal{C}_{2}, \nu_{\wp}, R_{\wp, 1+2 i}\right)$. We use the regions $U_{1+2 i}$ and $L$ constructed in Section 5.7.1]

Rotate the line $L$ shown in Figure $23 \pi / 2$ radians about the point $1 / 2+i / 2$ as instructed in Construction 2. This splits the region $U_{1+2 i}$ into four pieces (see Figure 28). These are the four sheets of the branched covering map $\wp^{2}$, and we obtain a region $Y$ which is isomorphic to the sphere.

We illustrate the partition $\mathcal{P}_{1+2 i}=\left\{A_{0}, A_{1}, A_{2}, A_{3}, A_{4}\right\}$ in Figure 29 An argument similar to the proof of Theorem [5.11)(3) gives that $\mathcal{P}_{1+2 i}$ is an independent generating partition.

5.7.3. The Elliptic Function $\left(\wp^{\prime}\right)^{2}$. Next, we examine rational maps which are factors of toral endomorphisms via the elliptic function $\left(\wp^{\prime}\right)^{2}$. Let $\rho=\frac{1+i \sqrt{3}}{2}$ and let $\Gamma_{\rho^{2}}=\left[1, \frac{-1+i \sqrt{3}}{2}\right]$. We define a rational map of the sphere by the equation $R_{\left(\wp^{\prime}\right)^{2}, \omega}(z)=\left(\wp^{\prime}\right)^{2} \circ T_{\omega} \circ\left(\left(\wp^{\prime}\right)^{2}\right)^{-1}(z)$. We define the measure $\nu_{\left(\wp^{\prime}\right)^{2}}(F)=$ $\operatorname{leb}\left(\left(\left(\wp^{\prime}\right)^{2}\right)^{-1}(F)\right)$ for all $F \in \mathcal{C}_{2}$.

The critical values of the rational map $R_{\left(\wp^{\prime}\right)^{2}, \omega}$ are

$$
e_{1}=\left(\wp^{\prime}\right)^{2}\left(\frac{1}{2}\right), \quad e_{2}=\left(\wp^{\prime}\right)^{2}\left(\frac{1}{2}+\frac{i \sqrt{3}}{6}\right), \text { and }\left(\wp^{\prime}\right)^{2}(0)=\infty .
$$

We note that the postcritical set of $R_{\left(\wp^{\prime}\right)^{2}, \omega}$ is $\left\{e_{1}, e_{2}, \infty\right\}$, hence none of the resulting rational maps $R_{\left(\wp^{\prime}\right)^{2}, \omega}$ are conjugate via a Möbius transformation to any in Section 5.7.1. We will need to examine the postcritical orbit or the degrees of the maps $R_{\left(\wp^{\prime}\right)^{2}, \omega}$ for each $\omega$ to determine if they are conformally conjugate to any of the rational maps in Section 5.7 .2

We illustrate $Y_{\left(\wp^{\prime}\right)^{2}}$ in Figure 30. We will use this particular subset of the period parallelogram $U_{\rho^{2}}$ for all of our discussion in this section. Let $\gamma_{j}=\rho^{j} Y_{\left(\wp^{\prime}\right)^{2}}$ where $j=0, \ldots, 5$. Then, $\bmod \Gamma_{\rho^{2}}$, we have that $\gamma_{j}$ and $\gamma_{k}$ intersect only on their boundaries for $k \neq j$, and $\bigcup \gamma_{j}=U_{\rho^{2}}$. Thus we see that for $\nu_{\left(\wp^{\prime}\right)^{2} \text {-almost every }}$ $z \in \mathbb{C}_{\infty}$ we have $\left.\wp^{2}\right|_{Y_{\left(\wp^{\prime}\right)}} ^{-1}(z)$ gives exactly one value in $Y_{\left(\wp^{\prime}\right)^{2}}$. Therefore, we obtain a well-defined map $T_{\left(\wp^{\prime}\right)^{2}, \omega}: Y_{\left(\wp^{\prime}\right)^{2}} \rightarrow Y_{\left(\wp^{\prime}\right)^{2}}$. 


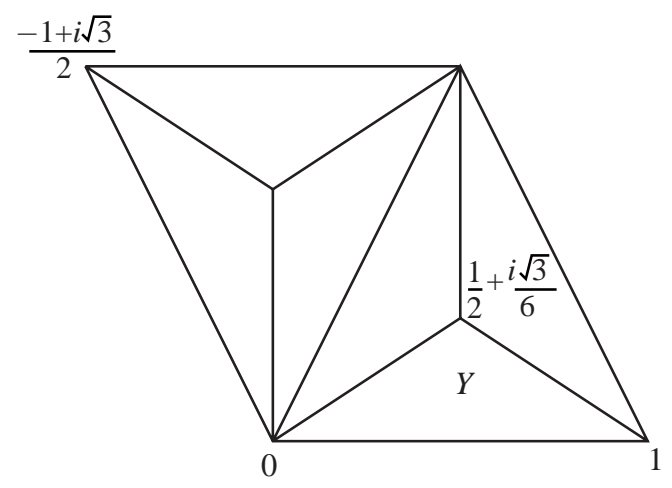

FIGURE 30. $Y_{\left(\wp^{\prime}\right)^{2}}$

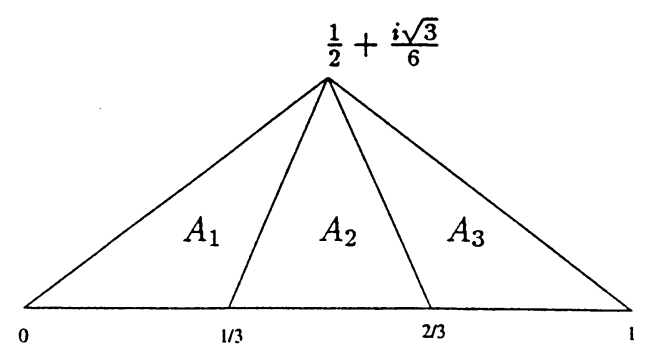

FIGURE $31 . \mathcal{P}_{i \sqrt{3}}$

Again, we use the appropriate $\sigma$-algebra and measure on $Y_{\left(\wp^{\prime}\right)^{2}}$ which make it a factor of $\mathbb{C} / \Gamma$. Let $\mathcal{C}$ be the Borel $\sigma$-algebra on $Y_{\left(\wp^{\prime}\right)^{2}}$ and $\mu_{\left(\wp^{\prime}\right)^{2}}=6$ leb $(E)$ for all $E \in \mathcal{C}$. Then $T_{\left(\wp^{\prime}\right)^{2}, \omega}$ is measure-preserving with respect to $\mu_{\left(\wp^{\prime}\right)^{2}}$. Again, we have the following reduction lemma.

Lemma 5.21. $\left(Y_{\left(\wp^{\prime}\right)^{2}}, \mathcal{C}_{2}, \mu_{\left(\wp^{\prime}\right)^{2}}, T_{\left(\wp^{\prime}\right)^{2}, \omega}\right) \cong\left(\mathbb{C}_{\infty}, \mathcal{C}_{2}, \nu_{\left(\wp^{\prime}\right)^{2}}, R_{\left(\wp^{\prime}\right)^{2}, \omega}\right)$.

Proof of Theorem $5.11(8)$. If $T(u)=i \sqrt{3} u$ is defined on the lattice $\Gamma_{\rho^{2}}$, then we obtain a degree three rational map of the sphere $R_{\left(\wp^{\prime}\right)^{2}, i \sqrt{3}}$. The postcritical set iterates in the following manner:

$$
\begin{gathered}
e_{1} \rightarrow e_{1} \ldots, \\
e_{2} \rightarrow \infty \rightarrow \infty \ldots,
\end{gathered}
$$

and hence $R_{\left(\wp^{\prime}\right)^{2}, i \sqrt{3}}$ is not conformally conjugate to any other map previously discussed in this chapter.

The partition $\mathcal{P}_{i \sqrt{3}}$ of $Y_{\left(\wp^{\prime}\right)^{2}}$ is shown in Figure [31. We include pictures of $\mathcal{P}_{i \sqrt{3}}^{1}$ and $\mathcal{P}_{i \sqrt{3}}^{2}$ in Figures 32 and 33 Each connected region has its own label. It is straightforward to show that $\mathcal{P}_{i \sqrt{3}}$ is an independent generating partition, and hence the theorem is proved.

Proof of Theorem 5.11(9). Continuing our examination of $(2,3,6)$ rational maps, we study the toral endomorphism $T_{1+i \sqrt{3}}(u)=(1+i \sqrt{3}) u$. Then $R_{\left(\wp^{\prime}\right)^{2}, 1+i \sqrt{3}}(z)=$ 


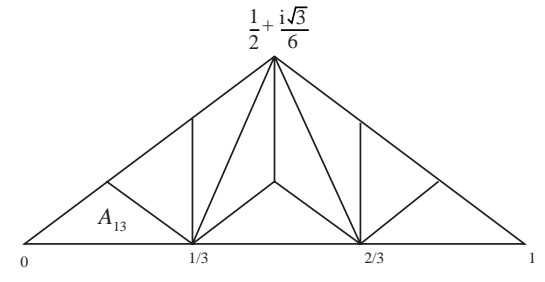

Figure $32 . \mathcal{P}_{i \sqrt{3}}^{1}$ and $\mathcal{P}_{3}$

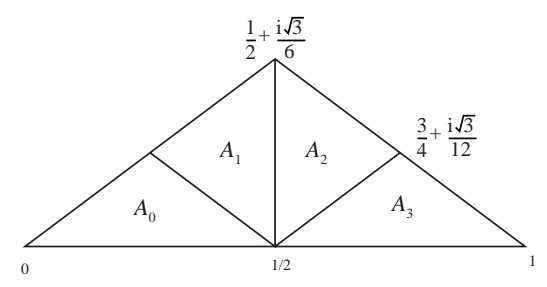

Figure $34 . \mathcal{P}_{1+i \sqrt{3}}$ and $\mathcal{P}_{2}$

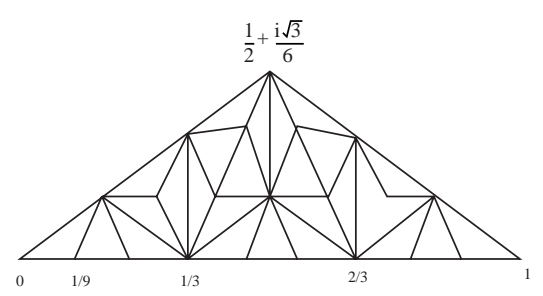

FIGURE $33 . \mathcal{P}_{i \sqrt{3}}^{2}$

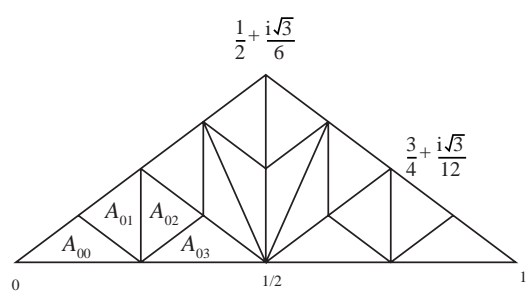

FiguRE $35 . \mathcal{P}_{2}^{1}$

$\left(\wp^{\prime}\right)^{2} \circ T_{1+i \sqrt{3}} \circ\left(\left(\wp^{\prime}\right)^{2}\right)^{-1}(z)$ gives a degree four map of the sphere. The postcritical orbit is described by

$$
\begin{gathered}
e_{2} \rightarrow e_{2} \ldots, \\
e_{1} \rightarrow \infty \rightarrow \infty \ldots .
\end{gathered}
$$

Each $A_{i}$ in the partition $\mathcal{P}_{1+i \sqrt{3}}$ shown in Figure 34 has area $\frac{\sqrt{3}}{48}$. The partition $\mathcal{P}_{1+i \sqrt{3}}$ is an independent generating partition with respect to $T_{\left(\wp^{\prime}\right)^{2}, 1+\sqrt{3}}$.

Proof of Theorem 5.11(10). Multiplication by an integer induces an endomorphism of any torus, and hence we obtain rational maps of the sphere as factors of toral endomorphisms $T_{2^{n}}(u)=2^{n} u$ via the elliptic function $\left(\wp^{\prime}\right)^{2}$ on $\mathbb{C} / \Gamma_{\rho^{2}}$. These maps have degree $2^{2 n}$ and the postcritical set behaves as follows:

$$
\begin{gathered}
e_{2} \rightarrow e_{2} \ldots, \\
e_{1} \rightarrow \infty \rightarrow \infty \ldots .
\end{gathered}
$$

When $n>2$ the degrees of these maps differ from those previously studied in this section. Further, the schematic diagram of the postcritical set differs from any map discussed in Sections 5.7.1 and 5.7.2. Thus these maps are not conformally conjugate to any others previously discussed in this paper.

Figure 34 illustrates a generating partition $\mathcal{P}_{2}$ for $T_{\left(\wp^{\prime}\right)^{2}, 2}$. Figure 35 shows the first refinement $\mathcal{P}_{2}^{1}$. The partition $\mathcal{P}_{2}^{n-1}$ is a generating partition for $T_{\left(\wp^{\prime}\right)^{2}, 2^{n}}$.

Proof of Theorem 5.11 (11). We can obtain rational maps of the sphere as a factor of the toral endomorphisms $T_{3^{n}}(u)=3^{n} u$ via the elliptic function $\left(\wp^{\prime}\right)^{2}$ on $\mathbb{C} / \Gamma_{\rho^{2}}$. These maps will have degree $3^{2 n}$ and the postcritical set behaves as follows:

$$
e_{1} \rightarrow e_{1} \ldots,
$$




$$
e_{2} \rightarrow \infty \rightarrow \infty \ldots
$$

When $n>1$ the degrees of these maps differ from those previously studied in this section. Further, the schematic diagram of the postcritical set differs from any map discussed in Sections 5.7.1 and 5.7.2. Thus these maps are not conformally conjugate to any others previously discussed in this thesis.

Figure 32 illustrates a partition of $Y$ into 9 atoms. We denote this partition by $\mathcal{P}_{3}\left(=\mathcal{P}_{i \sqrt{3}}^{1}\right)$. The partition $\mathcal{P}_{3}$ is independent and generates with respect to $T_{\left(\wp^{\prime}\right)^{2}, 3}$. It is straightforward to show that the partitions $\mathcal{P}_{3^{n}}=\mathcal{P}_{i \sqrt{3}}^{2 n-1}$ are independent generating partitions with respect to $T_{\left(\wp^{\prime}\right)^{2}, 3^{n}}$.

5.7.4. The Elliptic Function $\wp^{\prime}$. We proved in Lemma 5.2 that $\left(\mathbb{C}_{\infty}, \mathcal{C}_{2}, \nu_{\left(\wp^{\prime}\right)^{2}}\right.$, $\left.R_{\left(\wp^{\prime}\right)^{2}, \omega}\right)$ is a two-point factor of $\left(\mathbb{C}_{\infty}, \mathcal{C}_{2}, \nu_{\wp^{\prime}}, R_{\wp^{\prime}, \omega}\right)$. The results of Adler, Goodwyn, and Weiss $\mathrm{A}-\mathrm{G}-\mathrm{W}]$, Parry $[\mathrm{P}$, and the previous theorems of this paper give the following proof.

Proof of Theorem 5.11(12),(13),(14),(15). The maps given in this theorem are twopoint extensions of one-sided Bernoulli shifts. Thus the result follows from $\mathrm{A}-\mathrm{G}-\mathrm{W}$ and $[\mathrm{P}]$.

We note that the postcritical schematic diagrams that arise for the rational maps in cases (12), (13), (14), and (15) are identical to those which occurred in cases (10), $(11),(8)$, and (9) respectively.

After the first draft of this paper, the author received a preprint from D. Heicklen and C. Hoffman that rational maps of the sphere are one-sided Bernoulli with respect to the measure of maximal entropy.

\section{REFERENCES}

[A-L-W] J. Aaronson, M. Lin, and B. Weiss, Mixing properties of Markov operators and ergodic transformations, and ergodicity of Cartesian products, Israel J. Math. 33 (1979), 198 224. MR 81i:47010a

[A-G-W] R. Adler, L. Goodwyn, and B. Weiss, Equivalence of topological Markov shifts, Israel J. Math. 27 (1977), 49 - 63. MR 55:10639

[A-R] L.M. Abramov and V.A. Rokhlin, The entropy of a skew product of measure-preserving transformations, Amer. Math. Soc Transl. Ser. 2 48, 255 - 265.

[A-M-T] J. Ashley, B. Marcus, and S. Tuncel, The Classification of one-sided Markov chains, Erg. Th. Dyn. Sys. 17 (1997) 269 - 295. MR 98k:28021

[B] J. Barnes, Applications of noninvertible ergodic theory to rational maps of the sphere, Diss. Summ. Math. 1 (1996) 49 - 53. MR 98j:58095

[B-K] J. Barnes and L. Koss, One-sided Lebesgue Bernoulli maps of the sphere of degree $n^{2}$ and $2 n^{2}$, Internat. J. Math. Math. Sci. 23 (2000) 383 - 392. CMP 2000:12

[Be] A. Beardon, Iteration of Rational Functions, Graduate Texts in Mathematics, 132, Springer-Verlag, 1991. MR 92j:30026

[Be2] A. Beardon, The Geometry of Discrete Groups, Graduate Texts in Mathematics, 91, Springer-Verlag, 1983. MR 85d:22026

[Bo] L. Böettcher, The principal convergence laws for iterates and their applications to analysis, IZV. FIz.-Mat. Obshch. pri Imper. Kazanskom Univ. 13 (1903), no. 1, 1 - 37; 14 (1904), nos. 3-4, $155-234$.

[Bo] R. Bowen, Bernoulli equilibrium states for Axiom A diffeomorphisms, Math. Systems Theory 8 (1974/1975), 289 - 294. MR 52:8379

$[\mathrm{Br}-\mathrm{H}] \quad \mathrm{H}$. Bruin and J. Hawkins, Examples of expanding $C^{1}$ maps having no $\sigma$-finite measure equivalent to Lebesgue, Israel J. Math. 108 (1998) 83 - 107. MR 2000i:37051

[C-G] L. Carleson and T. Gamelin, Complex Dynamics, Univeristext Tracts in Mathematics, Springer-Verlag, 1993. MR 94h:30033 
[D-U] M. Denker and M. Urbanski, Ergodic theory of equilibrium states for rational maps, Nonlinearity 4 (1994), 103 - 134. MR 92a:58112

[D-H] A. Douady and J. Hubbard, A proof of Thurston's topological characterization of rational functions, Acta Math. 171 (1993), 263 - 297. MR 94j:58143

[E-H] S. Eigen and J. Hawkins, Examples and properties of nonexact ergodic shift measures, Indag. Math. (N.S.) 10 (1999), 25 - 44. MR 2000g:28036

[E-L] A.E. Eremenko and M. Yu. Lyubich, The dynamics of analytic transformations, Leningrad Math. J. 1 (1990), 563 - 634.

[F-L-M] A. Freire, A. Lopes, and R. Mañé, An invariant measure for rational maps, Bol. Soc. Brasil Mat. 14 (1993), 45 - 62. MR 91b:58109

[F] J.E. Fornaess, Dynamics in Several Complex Variables, C.B.M.S. Regional Conf. Ser. in Math. 87, 1996. MR 96j:32033

[F-S1] J.E. Fornaess and N. Sibony, Complex dynamics in higher dimension I, Astérisque 222 (1994), 201 - 231. MR 95i:32036

[F-S2] J.E. Fornaess and N. Sibony, Complex dynamics in higher dimension II, to appear in Ann. of Math. Stud., 137, Princeton Univ. Press, Princeton, NJ, 1995. MR 97g:32033

[G] M. Gromov, Entropy, homology and semialgebraic geometry (after Y. Yomdin), Seminaire Bourbaki Exposé 663 (1985), 225 - 240. MR 89f:58082

[H-P] J. Hubbard and P. Papadopol, Superattractive fixed points in $(C)^{n}$, Indiana Univ. Math. J. 43 (1994), 321 - 365. MR 95e:32025

[I] S. Ito, On the fractal curves induced from the complex radix expansion, Tokyo J. Math. 12 (1989), $299-320$.

[I-O] S. Ito and M. Ohtsuki, On the fractal curves induced from endomorphisms on a free group of rank 2, Tokyo J. Math. 14 (1991), 277 - 304. MR 92m:11078

[Ka] S. Kalikow, The T-T $T^{-1}$ transformation is not loosely Bernoulli, Ann. of Math. 115 (1982), 393 - 409. MR 85j:28019

[Ke] M. Keane, Strongly mixing g-measures, Invent. Math. 16 (1972), 309 - 324. MR 46:9295

[L] S. Lang, Elliptic Functions, Graduate Texts in Mathematics, 112, Springer-Verlag, 1987. MR 88c: 11028

[La] S. Làttes, Sur l'iteration des substitutions rationelles et les fonctions de Poincarè, C.R. Acad. Sci. Paris 166 Ser. 1 Math. (1919), 26 - 28.

[Ly1] M. Yu. Lyubich, The dynamics of rational transforms: the topological picture, Russian Math. Surveys 41 (1986), 43 - 117.

[Ly2] M. Yu. Lyubich, Entropy properties of rational endomorphisms of the Riemann sphere, Erg. Th. Dyn. Sys. 3 (1983), 351 - 385. MR 85k:58049

[M1] R. Mañé, On the uniqueness of the maximizing measure for rational maps, Bol. Soc. Brasil Mat. 14 (1983), 27 - 43. MR 85m:58110a

[M2] R. Mañé, Ergodic Theory and Differentiable Dynamics, Springer-Verlag, 1987. MR 88c:58040

[M3] R. Mañé, On the Bernoulli property for rational maps, Erg. Th. Dyn. Sys. 5 (1985), 71 - 88. MR 86i:58082

[Mc1] C. McMullen, Complex Dynamics and Renormalization, Princeton University Press, 1994. MR 96h:58097

[Mc2] C. McMullen, Families of rational maps and iterative root-finding algorithms, Ann. of Math. 125 (1987), 467 - 493. MR 88i:58082

[Mi] J. Milnor, Pasting together Julia sets - a worked out example of mating, preprint 1997.

[O] D. Ornstein, Factors of Bernoulli Shifts, Isr. J. Math 21(1975), 145 - 153. MR 52:3481

[P] W. Parry, Automorphisms of the Bernoulli endomorphism and a class of skew-products, Erg. Th. Dyn. Sys. 16 (1996), 519 - 529. MR 97h:28006

$[\mathrm{P}-\mathrm{T}] \quad$ W. Parry and S. Tuncel, Classification Problems in Ergodic Theory, Cambridge University Press, 1982. MR 84g:28024

[Pe] K. Petersen, Ergodic Theory, Cambridge University Press, 1983; corrected reprint, 1989. MR 87i:28002 MR 92c:28010

[R1] V.A. Rokhlin, Exact endomorphisms of a Lebesgue Space, Amer. Math. Soc. Transl. Ser. 239 (1964), 1-36.

[R2] V.A. Rokhlin, On the fundamental ideas of measure theory, Amer. Math. Soc. Transl. Ser. 171 (1952), 1-55. 
[Ru] D. Ruelle, Statistical mechanics of a one-dimensional lattice gas, Comm. Math. Phys. 9 (1968), 267 - 278. MR 38:3013

[T] W. Thurston, Lecture Notes, CBMS Conference, University of Minnesota at Duluth, 1983.

[U1] T. Ueda, Complex dynamical systems on projective spaces, Surikaisekikenkyusho Kokyuroka no. 814 (1992), 169 - 186. CMP 94:05

[U2] T. Ueda, Critical orbits of holomorphic maps on projective spaces, to appear in J. Geom. Anal. 8 (1998), 319 - 334. MR 2000f:32026

[W1] P. Walters, An Introduction to Ergodic Theory, Graduate Texts in Mathematics, 79, Springer-Verlag, 1982. MR 84e:28017

[W2] P. Walters, Invariant measures and equilibrium states for some mappings which expand distances, Trans. Amer. Math. Soc. 236 (1978), 121 - 153.

[W3] P. Walters, Some results on the classification of non-invertible measure-preserving transformations, Springer Lecture Notes in Math., Vol. 318, (1973), 266 - 276. MR 52:14234

[Z] A. Zdunik, Parabolic orbifolds and the dimension of the maximal measure for rational maps, Invent. Math. 99 (1990), 627 - 649. MR 90m:58120

Department of Mathematics and Computer Science, Dickinson College, P.O. Box 1773, Carlisle, Pennsylvania 17013

E-mail address: koss@dickinson.edu 